\title{
Filigrane
}

Écoutes psychothérapiques

\section{De la naissance de la psychanalyse à la naissance de la philosophie. Ou deux étonnantes métamorphoses d'Éros}

\section{Jean Bossé}

Volume 21, numéro 2, automne 2012

URI : https://id.erudit.org/iderudit/1015203ar

DOI : https://doi.org/10.7202/1015203ar

Aller au sommaire du numéro

Éditeur(s)

Revue Santé mentale au Québec

ISSN

1192-1412 (imprimé)

1911-4656 (numérique)

Découvrir la revue

Citer cet article

Bossé, J. (2012). De la naissance de la psychanalyse à la naissance de la philosophie. Ou deux étonnantes métamorphoses d’Éros. Filigrane, 21(2),

143-181. https://doi.org/10.7202/1015203ar
Résumé de l'article

Dans cet essai, l'auteur établit un parallélisme entre d'une part, la naissance de la philosophie comme discipline et l'importance que la relation Platon-Socrate y a jouée et, d'autre part, la naissance de la psychanalyse et la relation amoureuse (transfert) que fut celle de Freud à l'égard de Fliess. Ce faisant, il nous convie à une lecture attentive et renouvelée de leur célèbre correspondance et du Banquet de Platon. 


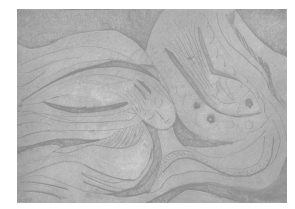

\title{
De la naissance de la psychanalyse à la naissance de la philosophie. Ou deux étonnantes métamorphoses d'Éros
}

\author{
Jean Bossé
}

Dans cet essai, l'auteur établit un parallélisme entre d'une part, la naissance de la philosophie comme discipline et l'importance que la relation Platon-Socrate y a jouée et, d'autre part, la naissance de la psychanalyse et la relation amoureuse (transfert) que fut celle de Freud à l'égard de Fliess. Ce faisant, il nous convie à une lecture attentive et renouvelée de leur célèbre correspondance et du Banquet de Platon.

À Roger Misès

Jamais le patient n'oublie ce qu'il a appris dans le transfert. Cette étonnante découverte a une force de conviction plus grande que tout ce qu'il peut acquérir par d'autres moyens.

S. Freud, L'Abrégé

On apprend de qui on aime.

Goethe

Être Alcibiade un jour et une nuit et mourir.

Goethe

D ès les premières lignes de la Métaphysique, Aristote affirme de façon péremptoire que tous les hommes ont par nature le désir de connaître. Mais comment se sont-ils mis à penser? D’abord, éblouis et émerveillés par le spectacle de la Nature et de l'Univers, ils les ont pour les comprendre longuement observés et ensuite, comme font tous les enfants devant la question de leur origine (d'où venons-nous?), ils ont commencé à inventer des mythes. Ceux-ci furent transmis en premier lieu oralement de bouche à 
oreille, d'une génération à l'autre, opération qui les a continuellement transformés.

Puis, vinrent les premiers poètes de la Grèce: Homère, Hésiode, Pindare, Tyrtée. Ceux-ci ont écrit des poèmes épiques qui racontaient ces mythes et du même souffle en proposaient de nouveaux. Leurs nombreux poèmes ont été lus et relus par tous les enfants d'Athènes puisqu' ils apprenaient à lire et à écrire en récitant par cœur les vers de ces grands poètes, en particulier ceux d'Homère.

Cependant, ces mythes ainsi transformés pendant des siècles et plusieurs générations, même s'ils leur donnaient une première réponse aux grandes questions que leur posait leur existence, les confinaient en même temps dans des modes de pensée qui n'étaient pas le leur. Ils ne leur permettaient pas d'atteindre une autonomie d'esprit et d'exercer leur capacité d'être seul.

Après un long moment de quelques siècles, il leur a fallu absolument sortir de cette dépendance aux dieux et rencontrer cette angoisse d'être seul qui est le sens réel du devenir humain : être soi-même par soi, en d'autres mots, comme le dira Socrate: "être un homme vraiment un homme». Pour cela, ils devaient développer coûte que coûte leur propre pensée. Mais ce mouvement de recherche ne pouvait s'accomplir qu'en passant obligatoirement par la connaissance de soi, c'est-à-dire de leur vie intérieure.

L'orientation de cette recherche particulière fut amorcée par Socrate au $\mathrm{V}^{\mathrm{e}}$ siècle av. J.-C. Nous allons retracer succinctement le moment historique de cet homme qui fut un être hors du commun. Mais avant, il est nécessaire d'exposer la situation politique qui s'est mise en place à partir du vi siècle av. J.-C. par l'action et la présence d'hommes politiques remarquables, tel Solon (640-558). Celui-ci, homme d'État et poète va établir les premiers éléments de base d'une future démocratie. Cette évolution sera continuée à la fin du $\mathrm{VI}^{\mathrm{e}}$ siècle par Clistène, jusqu'à la création de cette démocratie par Périclès (475-429). Ce dernier, à partir de 461 av. J.-C. fut élu stratège pendant trente années. Il organisa le fonctionnement de la première démocratie du monde qui restera en place de 461 jusqu'à 404, date de la défaite d'Athènes devant Sparte, après vingt-sept ans de guerre.

Ce siècle dit classique qu'on appelle communément aussi le siècle de Périclès fut marqué par la présence directe du peuple dans le pouvoir administratif de la cité et par l'élan et l'importance donnés : à l'éducation avec l'influence et l'enseignement de plusieurs sophistes; à la sculpture et à l'architecture et les constructions à Athènes avec Phidias; à la création théâtrale tragique avec Eschyle, Sophocle et Euripide; au théâtre comique avec Aristophane; et enfin à la peinture avec Zeuxis. 
C’est dans cette époque de grande créativité et de grande liberté de pensée que se situe le développement de la philosophie. Bien sûr, il y a eu avant Thalès et l'école de Milet, Héraclite, Démocrite, Pythagore, Parménide, Anaxagore, Protagoras ami de Périclès. Mais la philosophie comme discipline a vraiment commencé au Ve siècle avec Socrate et Platon. Elle a connu un essor considérable avec la fondation de l'Académie par Platon en 387 av. J.-C. dans l'antiquité et jusqu'à nos jours.

Mais comment est-elle venue à Socrate? Comment s'est-elle transmise à Platon? Voilà deux questions auxquelles je tenterai de répondre en prenant comme exemple la naissance de la psychanalyse et de Freud comme psychanalyste.

\section{L'origine de la psychanalyse}

Dans une étude antérieure sur ce sujet $(1993)^{1}$, en suivant pas à pas la relation Freud-Fliess, j'avais retracé l'origine de la psychanalyse et de la naissance de Freud comme psychanalyste. Pour une plus grande compréhension de cette démarche si singulière, je renvoie le lecteur à cette première étude publiée.

Pour bien saisir le parallélisme que je propose de faire entre, d'une part, la naissance de la philosophie comme discipline, et l'importance que la relation Platon-Socrate y a jouée et d'autre part, la naissance de la psychanalyse, il est nécessaire de rappeler succinctement les grandes lignes de cette relation amoureuse (transfert) que fut celle de Freud à l'égard de Fliess, et qui fut à l'origine de cette naissance.

Cette relation, amorcée en 1887, se terminera en 1902 par une rupture qui fut très douloureuse sans doute pour les deux. Quelle est la situation de Freud en ce moment particulier? Il vient de faire un stage à Paris dans le service du $\mathrm{P}^{r}$ Charcot, médecin éminent de l'hystérie. Immédiatement, Freud est saisi d'un réel envoûtement pour ce grand professeur ${ }^{2}$.

Manifestement, Freud est à la recherche d'un maître et prêt à vivre une relation de transfert. Grâce à l'admiration qu'il prêtait à Charcot, il s'est identifié aux hystériques de ce dernier : il s'y est reconnu ; premier pas important avant de prendre véritablement la position de patient de quelqu'un. Ainsi, il a pris conscience de sa propre folie. Mais Charcot n'est pas intéressé à ses recherches.

Revenu à Vienne en 1886, il reprend avec Breuer ses études sur l'hystérie et fait des hypothèses, mais celles-ci ne dépassent pas le point de vue médical. En somme, Breuer n'attend rien de Freud. Contrairement à celui-ci, Fliess 
s'intéresse à la sexualité, à son fonctionnement physiologique et biologique. De plus, il est lui-même demandeur: il a des ambitions scientifiques et il a besoin lui aussi de l'écoute d'un autre pour avancer.

Cette relation avec Fliess commence en 1887 et c'est Breuer qui les présente l'un à l'autre. À travers et par la relation transférentielle qui s'installera malgré lui (et dans la méconnaissance des deux), Freud va donc acquérir un autre «savoir », qui cette fois passe par l'inconscient et engage toute la personne. Voyons comment.

La trajectoire de cette relation n'est pas uniforme: comme toute relation amoureuse, elle suit de nombreux détours surprenants, imprévus et complexes. Elle durera une quinzaine d'années. Mais je retiendrai ici surtout les grandes lignes, «les temps forts» qui ont marqué l'évolution de Freud. On peut la diviser en deux grandes périodes.

\section{7-1895: séduction, symptôme et transfert}

D'emblée, Freud éprouve un véritable coup de foudre et place Fliess dans une grande surestimation ${ }^{3}$. Celle-ci apparait par l'absence de critique envers les théories de Fliess qu'il accepte presque aveuglément. La séduction réciproque est dès le début bien installée; ils se plaisent et ils rencontrent dans leur relation suffisamment d'affinité et d'attrait. Tout est probablement là pour que la séduction se rejoue; cependant, il faudra la méthode pour la regarder et Freud la trouvera pour lui-même dans l'étude de ses propres rêves.

Cette séduction est importante et elle est au cœur de la situation analytique (comme elle l'est, nous le verrons, dans le dialogue socratique); derrière le symptôme qui amène le patient en analyse se cache une demande de transfert. Elle est donc nécessaire à toute cure.

Et de quoi Freud et Fliess se parlent-ils? De leurs recherches, de leurs travaux, ils se font parvenir des publications et s'adressent des malades. Aussi, ils s'organisent des petits «congrès à deux». Mais, c'est Freud qui veut en savoir davantage. À partir de 1892, année où Fliess commence à publier, Freud écrit pour celui-ci. Il lui envoie des manuscrits qui sont des ébauches, des avancées théoriques sur les névroses et couvrant plusieurs sujets: de la névrose d'angoisse jusqu'à la paranoïa, en passant par l'hystérie, la mélancolie, la neurasthénie (dont il dit souffrir), les migraines (maladie de Fliess). À un certain niveau, ces manuscrits servaient probablement d'écran à la relation transférentielle qui s'installe de plus en plus profondément.

Pendant la même période, Freud rédige avec Breuer un ouvrage sur l'hystérie qui sera publié en 1895 sous le titre Les études sur l'hystérie. Au cours de 
cette préparation, la relation avec Breuer devient difficile. Breuer montre de la réticence devant l'hypothèse de l'origine sexuelle des névroses, idée centrale de Freud. Il commence par l'accepter et ensuite la rejette.

Bien entendu, cette volte-face de Breuer déçoit profondément Freud qui, dès lors, le désavouera comme «mentor». Pendant que leur amitié s'assombrit, le rapprochement avec Fliess se fait plus pressant et intense. L'intensification de la relation s'annonce d'ailleurs par une série de symptômes cardiaques: tachycardie, arythmie, douleurs thoraciques et dyspnée. Ce sont des symptômes annonciateurs d'une situation transférentielle «qui ne pouvait être que méconnue ${ }^{4}$ ». Écoutons Freud: «Je n'ai pas l'intention de m'étendre avec toi sur mon état cardiaque...» C'est le contraire qu'il faut entendre: «Je crois qu'il (le cœur) va de nouveau s'emporter dans les prochains jours ${ }^{5}$.»

Freud indique ici à Fliess, sans en être totalement conscient encore, que ses symptômes sont en rapport avec lui. Après tout, derrière ces malaises, n'est-ce pas d'une affaire de cœur dont il s'agit, et qu'il cherche à nier? Continuons la lecture de cette lettre: «Quelle tristesse pour un médecin qui consacre toutes les heures de la journée à l'étude des névroses, d'ignorer s'il est lui-même atteint d'une dépression raisonnablement motivée ou hypochondriaque. On a besoin d'être secouru ${ }^{6} . . . »$

Freud cherche naturellement à justifier et s'excuser de son «infidélité»: il a consulté Breuer pour son cœur. Fliess ne croit pas à l'origine psychogène et sexuelle de ses symptômes cardiaques, de ses propres céphalées et enfin, des névroses. C'est pourquoi Freud met en doute le diagnostic de Fliess (l'intoxication à la nicotine), il le fait vérifier par Breuer: «Cette fois, c'est de toi que je me méfie particulièrement, car je t'ai vu pour la première fois te contredire...»

La charge est ici assez violente. Il soupçonne Fliess de lui cacher la vérité, à savoir qu'il est atteint d'une maladie mortelle. Il est saisi d'une forte angoisse qui l'empêche d'avancer. L'intensité de sa passion amoureuse lui fait-elle craindre un danger de mort? Un mois plus tard (21-05-94), il expédie à Fliess une nouvelle série de manuscrits, l'un sur l'étiologie et la théorie des grandes névroses et un autre sur l'angoisse, "Comment naît l'angoisse ${ }^{7}$ ».

Comme nous le constatons, il cherche pour les deux. À la fin de sa lettre, il demande à Fliess «suggestions, compléments et éclaircissements », enfin il veut qu'il s'associe à lui plus intimement dans la compréhension de ses troubles. «Ainsi, nous rappelle Mannoni, Fliess était dans la position de médecin (Freud l'y mettait) et aussi, mais seulement à nos yeux de maintenant, 
également dans la position d'analyste. Comme analyste certes, il n'avait aucune compétence. On pourrait dire que comme médecin, il était tout aussi incompétent, mais c'est évidemment la même chose et une seule et même incompétence ${ }^{8}$.»

\section{5-1902: remémoration, répétition et perlaboration}

L'année 1895 apparaît comme une époque charnière dans l'analyse de Freud, ou mieux, un moment fort où il s'installe irréversiblement dans un processus analytique, mais lentement, après des intervalles de grande résistance et de souffrance. Cette évolution est provoquée par une suite d'événements qui vont heureusement entamer l'idéalisation du personnage Fliess. Celui-ci représente beaucoup de figures dans l'imaginaire de Freud: un mentor qui le précède et le guide, un témoin disponible, un bon public, un autre soi-même et enfin un confrère capable de le soigner quand il est malade. Cette idéalisation sera ébranlée, mais non le transfert, qui suivra son cheminement naturel. C'est grâce à cette "désidéalisation" que s'installe l'auto-analyse de Freud et qu'on voit poindre l'ambivalence qui fait partie de tout transfert. D'ailleurs, cette auto-analyse est un effet positif de la cure et marque un effort de l'analysé de prendre en main son propre processus et de faire seul une partie plus grande du travail ${ }^{9}$.

Déjà durant l'épisode cardiaque (1893-94), il avait douté de la perspicacité clinique de Fliess qui maintenait l'opinion d'une cause physique. Une première faille s'était ainsi créée; elle ira en s'élargissant sans cesse malgré de grands efforts pour colmater la brèche ouverte définitivement. À la fin du printemps 1895, Freud annonce à Fliess que Martha attend un sixième enfant et quatre semaines plus tard, il apprend de Fliess qu'Ida, sa femme, se trouve enceinte pour la première fois; réaction de déception et d'ironie à l'égard de Fliess, lui qui se disait sur le point de résoudre le problème de la contraception si importante pour Freud ${ }^{10}$.

Un dernier événement fut évidemment «l'affaire Emma», mise au jour par M. Schur en 1966. Freud avait en traitement Emma Eckstein, une amie de sa femme. Doutant de la nature hystérique de ses troubles abdominaux, il l'envoie donc à Fliess pour examen. Celui-ci suggère et fait une opération dans le nez. Mais par erreur et par acte manqué, il oublie $50 \mathrm{~cm}$ de gaze imprégnée de teinture d'iode; des suites opératoires graves entraînent infection et hémorragie et nécessitent une nouvelle intervention par un autre otorhino. La découverte de cet incident provoque une forte émotion chez Freud qui, comme il lui arrive en pareille occasion, a un léger évanouissement. Cette 
réaction psychosomatique semble liée à un sentiment de triomphe et à une forte «culpabilité de survivant» dont l'élaboration ne serait pas encore possible ${ }^{11}$.

On sait que Freud en fait part à Fliess qui réagit très mal; il se montre profondément blessé et exige une rétraction. Freud doublement embarrassé essaie de limiter les dégâts. Au fond de lui-même, il est certain que Fliess a tort, mais il est tellement désespéré à l'idée de le perdre ${ }^{12}$.

Évidemment, dans les circonstances, il pèche par excès. Ce trop laisse entrevoir son ambivalence; dans la même lettre, il conteste l'interprétation de Fliess sur l'origine nasale de ses troubles cardiaques: «En ce qui concerne mon mal, j'aimerais que tu aies raison de croire que le nez est pour beaucoup dans cela et que le cœur n'y est pour rien. Seul un juge très sévère me tiendra rigueur de ce que je crois le contraire... » Et un peu plus loin, il termine: "Ce que je voudrais finalement, c'est que tu acceptes de ne pas en savoir davantage sur cette question de cœur...» Il exprime par ces mots un désir de prendre en main son processus d'analyse.

C'est dans ce climat tendu qu'eut lieu dans la nuit du 23 au 24 juillet 1895 le rêve de l'Injection faite à Irma, premier grand rêve analysé par Freud. Au niveau de la relation transférentielle, il apparaît "comme un acte de répétition réparatrice post-traumatique; il revient sur l'incident relatif à Emma pour clore et barrer la route au doute quant à la valeur professionnelle et l'honnêteté morale de Fliess ${ }^{13}$ ». Il accomplit le désir de Freud de disculper Fliess et de ne pas compromettre sa relation, parce qu'au fond de lui-même, il sent qu'il a encore besoin de lui. Il n'en parle pas à Fliess, pourtant dès le lendemain il lui écrit mais, sans en souffler mot.

Les symptômes cardiaques disparaissent sans retour à la suite de l'interprétation de ce rêve. Que penser de l'acte manqué de Fliess? Une réponse qui s'adresse à Freud, identifié à une femme, Emma, par laquelle s'actualise un désir de rapprochement homosexuel. «Emma saigne et meurt aux yeux de Freud. Il s'évanouit, jamais il ne reviendra comme avant. Il a quelque peine à deviner la responsabilité de Fliess dans cette mutilation de femme, offerte comme objet transitionnel au désir homosexuel ${ }^{14}$. "La scène primitive de la psychanalyse est contenue et agie dans cette opération d'Emma. Il n'est pas anodin de rappeler ici que Freud, à la même occasion, va se faire opérer aussi par Fliess.

La même année, après la rencontre de septembre avec Fliess, il écrit le «grand projet» De l'esquisse d'une psychologie scientifique ${ }^{15}$ qu'il complète très rapidement; il l'envoie à Fliess et s'en désintéresse aussitôt. «Freud s'est 
jeté dans cette étude, comme nous le rappelle Mannoni, pour échapper à quelque chose qui concerne sa relation à Fliess. Mais il ne peut pas y échapper, la suite de son travail passe par elle ${ }^{16}$.»

La mort de son père, en 1896, va amener un grand remous et probablement, comme il le dira plus tard dans l'après-coup de la publication de L'interprétation des rêves, provoquer la nécessité incontournable d'entrer plus à fond dans sa propre analyse: le travail du deuil à faire. Le rêve et le travail sur le rêve sont travail de deuil, comme l'est au fond l'expérience de la cure. Cette mort précipitera la rédaction du grand livre sur les rêves, œuvre centrale et temps fort de son auto-analyse, son livre par excellence, celui sur lequel il reviendra constamment dans ses moments de doute.

Il semble, d'après les lettres à Fliess, que l'analyse intensive eut lieu surtout à la fin du printemps 1897; à partir de cette date, d'ailleurs, il n'envoie plus de manuscrit à Fliess. À la faveur de cette analyse, ses défenses cèdent et bientôt il peut traduire en termes clairs sa situation transférentielle: «Jamais je n'ai été atteint d'une paralysie intellectuelle pareille à la présente. Écrire la moindre ligne m'est un supplice... » Et plus loin dans cette lettre: «D'ailleurs, j'ai subi une sorte de névrose. Drôles d'états que le conscient ne semble saisir : pensées nébuleuses, doutes voilés, et à peine de temps en temps, un rayon lumineux. » Il termine en disant: «Il me semble être dans un cocon. Dieu sait quelle bête en sortira ${ }^{17} \ldots{ }^{\prime}$

Mais un mois plus tard, il est plus explicite sur son transfert. Retenons bien ses propos: «Je continue de ne pas savoir ce qui m’est arrivé. Quelque chose venu des profondeurs abyssales de ma propre névrose s'est opposé à ce que j'avance encore dans la compréhension des névroses. Et tu y étais, j'ignore pourquoi, impliqué. L'impossibilité d'écrire qui m'affecte semble avoir pour but de gêner nos relations. De tout cela je ne possède nulle preuve et il ne s'agit que d'impressions obscures... » Et, souhaitant une compréhension plus intime de la part de Fliess, il ajoute: «Quelque chose d'analogue s'est-il passé en toi ?» Autrement dit: Es-tu, toi-même, passé par là? Enfin, un peu plus loin, il revient à son intérêt et à son espace de certitude: «C'est l'explication des rêves qui me semble la plus certaine, mais tout autour se trouve une foule d'énigmes non encore résolues. Quant au côté organologique, c'est toi qu'il réclame ${ }^{18} \ldots »$

La lettre suivante annonce un pas de plus: «Celui de mes malades qui me préoccupe le plus c'est moi-même. Ma petite hystérie très aggravée par le travail s'est un peu atténuée. Le reste persiste encore et c'est de cela que dépend en premier lieu mon état d'âme. Cette analyse est plus malaisée que n'importe 
quelle autre et c'est elle aussi qui paralyse mon pouvoir d'exposer et de communiquer les notions acquises. Malgré tout, je crois qu'il faut la continuer et qu'elle constitue, dans mon travail, une indispensable pièce intermédiaire ${ }^{19}$...» Freud parle ici de sa maladie transférentielle comme une petite hystérie: nous sommes très près, en écoutant ce récit, de ce qu'il conceptualisera en 1914, Remémoration, répétition et perlaboration. Cette lettre annonce aussi un autre développement, et ce qui en lui fait obstacle: la découverte de l'importance des fantasmes inconscients et l'oedipe. Il lui faudra auparavant rejeter l'hypothèse de la séduction réelle, de la transgression sexuelle et des actes incestueux de la part du père, qui lui barre la route: « Je ne crois plus à ma neurotica...» L'œdipe fait son entrée très discrètement sous la forme d'une autre hypothèse: "C'est pourquoi une solution reste possible, elle est fournie par le fait que le fantasme sexuel se joue toujours autour du thème des parents ${ }^{20} \ldots$.. Freud fait une constatation capitale: la différence entre les fantasmes et les souvenirs réels.

Dans la lettre 70, quinze jours après, il montre clairement le lien entre les rêves et l'analyse, où il rejoint dans son souvenir, la séduction originaire; il l'avait attribuée à son père pour protéger sa «nania»: «1) Que dans mon cas, le père n'a joué aucun rôle actif, encore une analogie entre lui et moi; 2) Que ma "première génératrice» (de névrose) a été une femme âgée, laide et intelligente qui m'a parlé de Dieu et de l'enfer et m'a donné une haute idée de mes propres facultés. J'ai découvert aussi que, plus tard (entre 2 ans et 2 ans et demi), ma libido s'était éveillée et tournée vers matrem, cela à l'occasion d'un voyage de Leipzig à Vienne [...] au cours duquel je pus sans doute [...] la voir toute nue ${ }^{21}$.»

Il termine cette lettre le lendemain en parlant d'un rêve qui approfondit sa remémoration au sujet de sa bonne, son premier professeur de sexualité; il la met en rapport avec son professeur actuel, Fliess; cette séduction se rejoue donc ainsi dans le transfert. Enfin, il découvre que l'hypothèse du trauma a servi de résistance devant l'oedipe et son désir inconscient.

Douze jours plus tard, il écrit: «Mon auto-analyse est ce qu'il y a pour le moment de plus essentiel et promet d'avoir pour moi la plus grande importance si je parviens à l'achever. » Et plus loin, après avoir parlé longuement de souvenirs en questionnant sa mère, il poursuit: «Depuis, j’ai fait beaucoup de chemin mais sans avoir atteint encore mon point d'arrêt véritable. La narration de ce qui reste inachevé est si difficile, m'entraînerait si loin, que tu voudrais bien m'excuser et te contenter de l'exposé des parties bien vérifiées. Si l'analyse tient ce qu'elle promet, j'en coucherai systématiquement tous les 
détails et t'en soumettrai ensuite les résultats [...]» Il manifeste un désir, quoiqu'ambivalent, de tout dire et d'être sincère avec lui-même et avec celui qui lui tient lieu d'analyste. À la fin de cette lettre, on voit de nouveau poindre leurs différends, mis de côté momentanément par Freud: «Je m’intéresse si exclusivement à l'analyse que je n'ai pas encore essayé de mettre en parallèle nos deux hypothèses: la mienne suivant laquelle le refoulement émane toujours de la féminité et la tienne qui dit l'inverse ${ }^{22}$.»

Nous le retrouvons dans la lettre 75, à un moment important; après avoir exposé longuement sa théorie du refoulement et des névroses, il termine en avouant à Fliess la raison de sa résistance : «Mon auto-analyse reste toujours en plan. J'en ai maintenant compris les raisons. C'est parce que je ne puis m'analyser moi-même qu'en me servant de connaissances acquises (comme pour un étranger). Une vraie auto-analyse est réellement impossible, sans quoi il n'y aurait pas de maladie ${ }^{23} . . . »$ Freud ici admet (il en a manifestement l'intuition) l'importance essentielle de l'autre pour avancer et nous verrons qu'il prendra beaucoup de temps à en tirer toutes les conséquences. «L'autoanalyse est possible, dit O. Mannoni, puisqu'on s'analyse comme un autre et, pour la même raison, elle n'est pas une auto-analyse. C'est Freud lui-même et non Fliess qui s'est analysé comme un autre - et en cela, son travail avec ses patients a joué un rôle qui semble très important. Mais c'est Fliess qui à son insu l'a mis dans une situation transférentielle, où le savoir se modifie dans ses rapports avec l'inconscient. » À la faveur de cette rencontre, il accomplit une «mutation décisive ». «Ce qu'il avait appris chez Charcot, c'était à s’identifier au patient. Ce qu'il a appris de Breuer, c'est que Breuer ne savait rien d'autre que ce que sa patiente pouvait lui apprendre. Et ce qu'il avait à apprendre de Fliess, c'est que le patient apprend tout l'essentiel du transfert luimême ${ }^{24}$.»

Ceci est vrai, mais demande un certain développement, en tout cas des précisions que ne manque pas d'apporter Laplanche dans sa critique: «Qu’il l'appelle Selbstanalyse, analyse de soi, n'entraîne en rien qu'il s'agit d'un processus analytique [...] Toute prétendue analyse se situe dans l'adresse à quelqu'un, et on doit pouvoir y repérer le transfert ${ }^{25}$.» Tout comme bien entendu l'auto-analyse qui naît dans l'analyse et se poursuit après la terminaison, ne s'adresse-t-elle pas toujours en fin de compte à l'ex-analyste, comme «le destinataire» ou «le messager disparu», pour autant que cette relation transférentielle reste forcément un lieu, un creuset d'élaboration, ou mieux de perlaboration (ducharbeiten) ? Il ne suffit pas simplement d'énoncer le symptôme ou le transfert pour l'élucider, mais il importe de le perlaborer, 
c'est-à-dire de le travailler en le traversant, trouver les significations qui maintiennent les mécanismes répétitifs.

\section{Rupture et mort d'une amitié}

Avec la fin de l'année 1897, nous entrons dans la phase terminale de leur relation. À la suite du « congrès » de Breslau où Fliess a entretenu Freud de sa théorie de la bilatéralité, la lettre suivante fait allusion à leurs divergences. La réaction de Fliess est de nouveau ombrageuse. Freud s'explique sur la bisexualité et la bilatéralité, et ajoute un commentaire significatif: «Tu me connais assez bien et depuis assez longtemps pour t'en prendre à toi-même, s'il te reste des choses intimes à apprendre de moi $^{26}$.»

Comment comprendre cette affirmation? D'une part, il fait de son mieux pour amadouer Fliess et apaiser sa colère. D'autre part, Freud ne ditil pas de façon claire que de son côté, il a essayé de tout lui révéler? Écoutons Freud encore: "J'abandonne l'auto-analyse pour me consacrer au livre sur les rêves ${ }^{27}$.» Est-il en proie à une résistance concernant son analyse? Ou est-il simplement pris de court par la réaction de Fliess vis-àvis ses objections et du fait qu'il n'est pas d'accord avec lui et qu'il ne se sent pas capable encore de se passer entièrement de lui ? Grâce à cette relation et à partir de la reconstruction de son enfance, Freud a réalisé et rapporté à Fliess ses découvertes les plus extraordinaires: le sens des rêves, le complexe d'Edipe, la scène primitive et l'angoisse de castration. De plus, toute une série de rêves que Freud classera dans la rubrique des « rêves absurdes» apparaissent dans l'année 98: Mon Fils, Le Myope, La Monographie Botanique, Non Vixit, Goethe attaque M. M., Le Comte de Thun. Ces rêves, comme l'a bien noté Anzieu ${ }^{28}$, poursuivent le dégagement de Freud par rapport à l'image toute-puissante du père, projetée sur Fliess. Sur le plan réel, il met d'ailleurs plus directement en question les idées de Fliess et enfin sa relation transférentielle.

En même temps, les lettres subissent un ralentissement, une fermeture dans la liberté d'écriture; dans les lettres 84 à 101, il y a peu d'allusions à l'auto-analyse et la rédaction du livre sur les rêves est en panne. De fait, il se sent «l'esprit paralysé» vis-à-vis du problème de la métapsychologie, le grand chapitre VII ; sans doute, a-t-il besoin d'un plus large dégagement transférentiel pour réaliser cette étape majeure. Malheureusement, elle ne se fera pas sans heurt. Par son histoire infantile et ses premières expériences objectales, pressent-il dans son inconscient que ses objets sont très vulnérables à ses propres attaques et sa destructivité? 
La lecture des lettres 101 à 152 est révélatrice de ce mouvement d'autonomie de Freud et aussi de l'échec de ce dégagement psychique nécessaire; un échec surtout dû à la réaction d'opposition de la part de Fliess. Relisons-les attentivement.

Freud émet (lettres 101 et 102) des doutes sérieux sur la théorie des périodes de Fliess; en fait, il craint beaucoup de le voir négliger les facteurs dynamiques du psychisme. Mais il demeure très préoccupé de la santé de Fliess, probablement quant à sa capacité de tenir le coup devant lui. Il lui expose une théorie sur ses maux de tête. Au fond, c'est une interprétation sur ses malaises qu'il lui donne; elle concerne le déplacement vers le haut de l'hystérique et l'origine sexuelle de ce trouble lui semble maintenant de plus en plus évidente. Dans les lettres suivantes (113 à 116), il est question de la rédaction finale de L'interprétation des rêves que Freud donne à lire à Fliess qui demande d'enlever un certain rêve: est-il trop intime ou sexuel, ou trop transférentiel?

Deux passages de la lettre 119 nous font pressentir une nouvelle intensification du conflit, dans la mesure où la publication du «livre sur les rêves» marque une étape majeure pour Freud, comme prise en main de son destin et de sa personne et comme transgression inévitable. Les rêves de Rome (janvier 97) sont à ce sujet très révélateurs. «Tu l'auras senti comme moi et nous avons toujours été francs l'un envers l'autre pour nous jeter mutuellement de la poudre aux yeux. C'est dans l'impossibilité de faire mieux que je trouve une consolation. Je suis désolé aussi de devoir perdre la bienveillance du plus cher et du meilleur de mes lecteurs en lui communiquant les épreuves, car comment trouver bien les épreuves d'un ouvrage que l'on se voit obligé de corriger ${ }^{29}$ ? » Surtout quand elle contient tant d'indices de son ambivalence, en particulier de sa haine, de ses souhaits de mort. D’ailleurs, les dernières lignes y font spécifiquement allusion, en parlant des rêves absurdes: "Il est étonnant de voir avec quelle fréquence tu y apparais. Dans le rêve non vixit, je me réjouis de t'avoir survécu. N'est-il pas terrible de devoir avouer une chose semblable, à une personne qui peut l'interpréter ${ }^{30}$ ?»

Le livre est envoyé à Fliess comme cadeau d'anniversaire: Freud reconnaissant, par son geste à ce dernier, le rôle qu'il a joué et l'importance qu'il eut dans la naissance de cet enfant, de la psychanalyse, et de lui-même comme psychanalyste. Il est évident aussi que son rôle n'est pas terminé; il insiste particulièrement sur celui-ci dans la lettre 122 en disant qu'il lui «faudra aussi une forte impulsion venant du côté de l'amitié ${ }^{31} »$.

À partir du moment où «le livre des rêves» est publié, Freud revient au transfert, mais annonce une dernière étape: après avoir éprouvé aussi 
intensément durant ces derniers mois ce grand besoin de vivre près de lui et avoir traversé une crise intérieure si profonde, il n'a pas l'intention d'accepter son invitation de se rencontrer à Pâques. «En réalité, tout permet de croire que je veux t'éviter». Un peu plus loin: «mais, il y a d'autres motifs intérieurs, faits d'un agrégat d'impondérables qui pèsent lourdement sur moi... Je me sens intérieurement très appauvri. J'ai été obligé de démolir tous mes châteaux en Espagne et c'est maintenant que je récupère un peu de courage pour les reconstruire. Tu m'aurais été un inappréciable secours pendant cet écroulement catastrophique; au stade actuel, je serais incapable de me faire comprendre de toi ${ }^{32} .$. » Lettre combien émouvante; on saisit clairement une mutation importante et la nécessité pour Freud de faire face seul à son destin.

Du reste, dans la lettre 134, on note un pas de plus dans son deuil et l'acceptation de la distance: «Je n'ai rien à objecter au fait du splendide isolement, s'il ne s'étendait trop loin et même jusqu'à nous deux. Dans l'ensemble - en dehors de mon point faible, la peur de la pauvreté - je suis trop raisonnable pour me plaindre et me sens d'ailleurs actuellement trop bien pour cela. [...] Mais rien ne peut pour moi remplacer les contacts avec un ami, c'est un besoin qui répond à quelque chose de féminin ${ }^{33} .$. » Et à peine un mois plus tard se manifeste un sursaut nostalgique pour récupérer l'objet: «Je voudrais m'enterrer quelques semaines dans un endroit où il ne serait pas question de sciences, sauf en ce qui concerne mes congrès avec toi ${ }^{34}$.

Deux mois après (été 1900), encore secoué par l'échec du «livre des rêves », il écrit : «En ce qui concerne les grands problèmes, rien n'est encore décidé. Tout est flottant, vague, un enfer intellectuel, des couches superposées, et dans les tréfonds ténébreux se distingue la silhouette de Lucifer-Amor ${ }^{35}$.» Cette lettre est la dernière avant leur ultime rencontre à Achensee, qui fut très orageuse; les différends, jusque là latents, sont devenus très manifestes.

«L'air est maintenant tout rempli d'un tel fantôme, qu'aucun ne sait comment l'éviter.» (Goethe)

À partir de cette date, nous entrons dans une période aiguë de leurs conflits, où la relation va se détériorer rapidement et évoluer vers une rupture irrémédiable: «Pas plus que toi je n’irai à Rome (allusion à la lettre 136) [...] Je savais très bien qu'un tel rappel serait, justement à cette heure, fort déplacé. Je ne faisais que fuir devant le présent dans la plus belle de mes fantaisies d'alors, je me rendais bien compte dans laquelle. Entre-temps, les congrès eux-mêmes sont devenus des survivances du passé. Je ne fais rien de nouveau, et je suis devenu, comme tu me l'écris, entièrement étranger à ce que tu fais toi-même ${ }^{36} \ldots$ » 
On pourrait considérer cette affirmation de Freud comme une interprétation de rejet à l'égard de Fliess. Dans celle-ci, il fait référence aux théories des périodes et de la bilatéralité sur lesquelles il n'est plus d'accord. Ce désinvestissement touche profondément Fliess qui a encore besoin de Freud. De plus, l'oubli de ce dernier concernant la priorité de la bisexualité est certainement une autre manifestation de rejet. Mais la rupture définitive sera provoquée par deux interprétations (sauvages?) de Fliess; celles-ci font narcissiquement mal à Freud. De prime abord, elles apparaissent comme des réactions d'irritation et de colère envers Freud, qui éprouve un besoin profond de distanciation et de détachement.

Écoutons Freud dans sa lettre 145, lettre mémorable et capitale pour comprendre la nature de leurs conflits relationnels: «Il est impossible de nous dissimuler que, toi et moi, nous nous sommes éloignés l'un de l'autre; toutes sortes de petites choses me le font voir... Tu atteins là les limites de la perspicacité. Tu prends parti contre moi en disant que "celui qui lit la pensée d'autrui n'y trouve que ses propres pensées", ce qui ôte toute valeur à ma recherche.»

Freud réagit très fortement à cette première interprétation: « S'il en est ainsi, jette sans la lire, ma Psychopathologie dans la corbeille à papier. Il y a dans ce livre des tas de choses qui te concernent pour lesquelles tu m'as fourni des matériaux et des choses cachées dont la motivation t'est due. Tu m'as aussi fourni l'épigraphe. Quelle que soit la valeur durable de cet ouvrage, tu y trouveras la preuve du rôle que tu as, jusqu'à présent, tenu dans ma vie. Après une telle déclaration, j'ai sans doute le droit de t'envoyer mon livre, dès que je l'aurai entre les mains, sans rien ajouter de plus ${ }^{37}$.»

Un passage inédit de cette lettre (cité par Schur) nous livre une autre interprétation de Fliess qui s'adresse à Breuer, mais Freud y voit un jugement sur sa propre amitié, sur ses sentiments envers Fliess: «En ce qui concerne Breuer, tu as certainement tout à fait raison de l'appeler le frère. Toutefois, je ne partage pas ton mépris pour l'amitié masculine, sans doute parce que cela me touche de trop près. Comme tu le sais, la femme n'a jamais remplacé dans ma vie le camarade, l'ami. Si l'évolution masculine de Breuer n'était pas si tordue, si timide, si contradictoire, comme tout ce qui touche à l'âme chez lui, il serait un bon exemple des exploits vers lesquels peut être sublimé, chez l'homme, le courant androphile ${ }^{38} \ldots$...

Cependant, Freud répond à Fliess : toi aussi tu méprises mon amitié, alors tu seras aussi écarté. En rejetant son ami qui était l'autre dans le transfert, Freud jette le bébé avec l'eau du bain, comme nous allons le constater. Il ne 
veut plus de Fliess comme figure transférentielle quoiqu'il ait encore besoin de cet autre pour élaborer un dernier point fondamental, un dernier bastion de son analyse: sa bisexualité, son homosexualité, «là où le paranoïaque échoue. » C'est d'ailleurs sur ce thème qu'il terminera son œuvre, 35 ans plus tard, dans son travail L'analyse avec fin et l'analyse sans fin $^{39}$.

Cette rupture, avec tout ce qu'elle représentait pour Freud, maintiendra au fond de lui-même une profonde douleur et rejoindra les autres traumatismes vécus dans son enfance avec ses objets premiers. Il restera marqué toute sa vie par cette impossibilité: celle de ne pouvoir peut-être se donner une occasion de réconciliation réelle. À la place subsisteront une culpabilité profonde et des causes dépressives. D'ailleurs, cette impasse n'a pas échappé à M. Schur qui y a vu une conséquence grave: "On sait qu'un des facteurs importants de cette phase (terminale) est la dissolution progressive de la relation transférentielle; le processus soumis à une analyse constante ne doit pas être bouleversé par un conflit résultant d'événements extérieurs. Si cette phase n'est pas harmonieuse, les conflits non résolus persistent fréquemment et sont durables. Or, la fin de la relation Freud-Fliess fut rien de moins qu'harmonieuse ${ }^{40}$.»

Sur le plan plus proprement analytique, Freud a plutôt interrompu cette relation, sans en avoir terminé psychiquement. Que pouvait-il en attendre encore? Dans l'après-coup nous pourrions répondre: la présence bienveillante d'un autre qui lui aurait permis d'explorer jusqu'au bout son processus concernant le problème de son homosexualité latente et d'y voir son aspect positif, la reconnaissance de la bisexualité psychique et le meurtre de l'objet transférentiel au niveau symbolique; un autre donc qui aurait accepté d'être détruit/créé, et ainsi de survivre pour que l'identification à la fonction de l'autre (l'analyste) puisse se faire ${ }^{41}$. Je reviendrai sur l'importance de ce courant homosexuel positif de la relation maître-élève dans la deuxième partie de ce texte.

Cette fin malheureuse de la relation Freud-Fliess a eu des conséquences aussi dans la transmission de la psychanalyse. Freud a mis beaucoup de temps à reconnaître l'importance du transfert dans la situation thérapeutique, le levier indispensable qu'il représentait dans l'évolution du patient et dans la transmission de la psychanalyse, c'est-à-dire l'enseignement et la formation des premiers analystes. Au début, on le voit même se fâcher contre les élèves qui voulaient entreprendre une cure avec lui. Plusieurs analyses des premiers disciples ont été épistolaires. Redoutait-il l'impasse qu'il avait rencontrée dans la sienne? Il voulait probablement éviter le problème de l'homosexualité latente qui ne manque jamais de se manifester. 
Malgré sa situation difficile, plus particulièrement son achoppement dans sa phase terminale, l'expérience de Freud dans l'ensemble est loin d'avoir été négative. Il nous a laissé une œuvre écrite colossale ${ }^{42}$, fruit de son élaboration psychique, là où s'exprima son immense génie. Par cette œuvre, il nous a donné l'essentiel: la méthode analytique et la mise en place d'un certain nombre de concepts fondamentaux qui représentent l'ensemble le plus cohérent et la compréhension la plus profonde qui soit du fonctionnement psychique. Par sa référence permanente, elle continue de nous faire élaborer en tant qu'analyste et, in petto, nous la travaillons aussi à notre tour; dans notre appropriation de l'inconscient, nous la faisons progresser dans des régions inexplorées, et sur des questions simplement indiquées et laissées en plan par Freud.

Maintenant que nous avons saisi comment sont nés la psychanalyse et Freud comme psychanalyste, regardons ce qui s'est passé pour la philosophie antique.

\section{Naissance de la philosophie antique et naissance de Platon comme philosophe}

\section{La figure de Socrate}

Nous savons peu de choses de Socrate lui-même puisqu'il n'a rien écrit. La plupart des notices biographiques que nous possédons, nous les devons à quatre sources principales: 1) à Platon tout d'abord qui l'a mis au centre de ses dialogues, au nombre de 35 environ, 2) à Xénophon, qui nous en parle dans ses Mémorables, son Apologie, son Banquet, 3) à Aristote duquel nous avons des allusions ou citations (une quarantaine environ) parsemées dans ses écrits, 4) et enfin à Aristophane, auteur comique du ve siècle av. J.-C. ; dans les Nuées et les Grenouilles où il le met particulièrement en scène, surtout en le ridiculisant.

Cependant, dans le présent travail, je m'attacherai surtout au Socrate tel que présenté et figuré par Platon: "Socrate qui naît du travail de Platon, fécondé lui-même par Socrate ${ }^{43}$.» C'est précisément la relation Platon-Socrate qui me servira pour élaborer la question de la naissance de la philosophie antique: comment la philosophie est-elle venue à Platon, disciple de Socrate? Et aussi comment s'est-elle transmise? Cette méthode de transmission fut probablement mise en place au moment de la fondation de l'Académie par Platon en 385 av. J.-C.; celle-ci fut le haut lieu de l'enseignement de la philosophie et de la formation des philosophes. À partir du IV siècle av. J.-C., elle sera donc le premier centre universitaire de l'Occident ${ }^{44}$. 


\section{Quelques lambeaux historiques de Socrate}

Socrate est né vers 470 av. J.-C., d'un père sculpteur (ou tailleur de pierre), Sophronisque et d'une mère, sage-femme, Phénarète, du dème (bourg) d'Alopèce. Issu d'un milieu simple et artisan, il a probablement eu une éducation de base, celle qu'habituellement recevaient les jeunes Athéniens de son époque. Selon certains commentateurs, il aurait été mis en apprentissage de la sculpture, comme il était coutume en Grèce antique pour les fils d'embrasser le métier de leur père.

Il aurait servi comme hoplite dans trois guerres : la campagne de Potidée, en 432-429, où il sauva Alcibiade blessé en le protégeant et le transportant sur ses épaules; la campagne de Delium, en 424, au cours de laquelle il sauva Xénophon; et enfin la bataille d'Amphipolis (en Macédoine) en 422.

Socrate aurait, semble-t-il, connu des maîtres de loin: Héraclite et Parménide qu'il aurait lus sur la recommandation d'Euripide. De plus près, il aurait connu Anaxagore, Archélaos et Protagoras qui étaient dans l'entourage de Périclès; puis certains autres sophistes: Prodicos, Hippias, Gorgias et Antiphon le sophiste. Ces derniers, malgré la critique négative de Socrate (ou de Platon) ont été d'excellents professeurs qui ont apporté beaucoup à la Grèce antique ${ }^{45}$. Il y avait parmi eux de bons philosophes, témoignant d'une grande liberté d'esprit. Quelques-uns ont connu le sort de Socrate, tels Anaxagore (?), Antiphon et Protagoras.

Plusieurs historiens affirment que Socrate a voulu avant tout se démarquer des sophistes (est-ce encore le désir de Platon?). Ils ont reconnu en lui l'intention avouée de faire table rase de ses prédécesseurs ${ }^{46}$. Quelques-uns (tels Diogène de Laërce et Aristote) ont noté chez lui un moment historique important à la suite d'une visite qu'il fit à Delphes. À cette occasion, il découvre la célèbre recommandation du dieu Apollon : «Connais-toi toi-même» inscrite sur le fronton du temple. À partir de cet instant, Socrate se voit assigner par le dieu une mission dont il ne se départira jamais jusqu'à sa mort, malgré les menaces qui lui furent adressées. Quelle était cette mission? Elle visait avant tout à rendre meilleurs lui-même et les autres, sous le thème de "l'arêtè», c'est-à-dire de la vertu, de la dignité, de l'excellence de l'âme et, par là, se réformer lui-même et les autres afin de procurer le bonheur à la cité. Pour arriver à cette fin, Socrate propose la réflexion et le dialogue soutenus par sa maïeutique: une méthode d'investigation, ou mieux d'interrogation et de réfutation, laquelle conduit lui-même et les autres à prendre conscience de leur ignorance, plus précisément de leur non-savoir et de leur non-sagesse. Cette méthode amène donc à une meilleure connaissance de soi et surtout de son âme. 
Socrate adopte l'attitude naïve de «celui qui ne sait rien», expression de la fameuse « ironie socratique», de l'ignorance feinte ${ }^{47}$. Ce qu'il propose est une profonde "révolution du concept du savoir ${ }^{48}$ ».

Cette figure de Socrate sera remarquablement illustrée par le génie de Platon, génie littéraire d'exposition théâtrale et dramatique, dans ses célèbres dialogues. Ceux-ci constituent la somme de l'œuvre de Platon ainsi parvenue jusqu'à nous.

\section{Les débuts de la relation Platon-Socrate}

Platon devint un familier de Socrate vers 408-407 av. J.-C., et il le sera jusqu'à la mort de celui-ci en 399 av. J.-C., après un procès retentissant pour impiété où il fut condamné à mort. Comme nous allons le montrer, tout ce drame fut un événement central et tragique dans la vie de Platon. Il nous en parle dans ses premiers dialogues dits socratiques: Apologie de Socrate, Criton, Euthyphron, Phédon. Je reviendrai plus loin sur l'importance de ce procès et de la mort de Socrate dans l'évolution personnelle de Platon.

\section{Divers éléments historiques concernant Platon}

Comme pour Socrate, nous avons peu d'informations sur l'histoire personnelle de Platon. Notons qu'il fut un enfant de la guerre ${ }^{49}$. Il est le quatrième enfant d'une famille aristocratique qui gravite près du pouvoir. Sa mère, Périctioné serait une descendante de Solon, l'un des sept sages de la Grèce, dont nous avons parlé succinctement plus haut. Son père, Ariston, descendait aussi d'une autre famille célèbre, celle du roi Codros, dernier roi d'Athènes. Ce père meurt à sa naissance en 428-427 av. J.-C., ou à une date tout près de celle-ci et de la peste qui sévit à Athènes entre 429-27, laquelle emporta d'ailleurs Périclès et ses deux fils. Platon n'a donc pas connu son père. Sa mère se remariera un peu plus tard alors que Platon était près de l'adolescence (?) ${ }^{50}$, avec Pérylampe, ami de Périclès et oncle du côté maternel, auquel elle donnera un autre fils, Antiphon. Platon se serait très mal entendu avec ce nouveau conjoint, qui aurait été d'une grande violence.

Comment fut comblée cette absence de son père dans son développement? On parle de son professeur de lutte, Phidias (?) ${ }^{51}$ qui lui donna le nom de Platon du fait de la largeur de son front ou de ses épaules, alors qu'il aurait dû porter le nom de son grand-père, Aristoclès. Critias, un cousin de sa mère qui pendant un temps très court fut disciple de Socrate, s'occupa de lui durant l'adolescence. Il l'initia à la poésie, à l'écriture, à la philosophie et à la politique. Ce même Critias devint membre du Gouvernement des Trente (ou 
Trente Tyrans) imposé à Athènes par Sparte après la défaite en 404. Dès qu'il fut en fonction, ce gouvernement, en particulier Critias, devint très répressif voire sanguinaire vis-à-vis la population d'Athènes. Cette attitude de Critias dégoûta Platon à jamais de l'exercice du pouvoir et l'éloigna d'une carrière politique à laquelle, semble-t-il, il se destinait ${ }^{52}$.

\section{Rencontre de Platon avec Socrate}

À sa première rencontre avec Socrate en 408-407 av. J.-C., Platon, âgé alors d'une vingtaine d'années, fut complètement envoûté et séduit par le personnage et par tout ce qu'il proposait aux jeunes Athéniens. Il réagit en jetant au feu tous ses manuscrits de poésie et de tragédies qu'il avait déjà écrits. "Quand je lui prête l'oreille, mon cour bat plus fort que celui des Corybantes et ses paroles me tirent des larmes ${ }^{53}$.» Après cette tirade amoureuse qu'il faire dire à Alcibiade, il est permis d'imaginer l'importance et la profondeur de cette relation amoureuse qui s'est tissée durant leurs huit années de fréquentation continue. Car, ce n'est pas seulement une façon de penser que proposait Socrate à tous ces jeunes disciples athéniens mais un concept de philosophie conçu comme un mode de vie ${ }^{54}$. De plus, à ce moment de sa vie où Platon était à la croisée des chemins et en réflexion sur son avenir, cette rencontre avec Socrate nous apparaît déterminante. Peutêtre était-il à la recherche d'un maître, voire un passeur indispensable pour franchir cette étape de sa vie: trouver sa voie?

Quel drame, quelle blessure, quelle indignation et quel immense chagrin dût-il éprouver à l'annonce de ce procès pour impiété qu'on intenta à Socrate. D’autant que les accusations portées - corrompre la jeunesse, ne pas reconnaître les dieux d'Athènes et en proposer d'autres - paraissaient si fausses et si arbitraires à l'égard de «l'homme le meilleur et le plus juste parmi les hommes».

Ceci arriva en 399 av. J.-C., au moment où précisément un gouvernement démocratique était revenu au pouvoir après quatre années de guerre civile. Paradoxalement, c'est ce gouvernement qui par ses juges, va commettre le plus infâme des assassinats, celui de son maître bien-aimé, ce "père libérateur» (L. A. Dorion). De plus, cette condamnation était d'autant plus cruelle pour Platon qui, lors du procès, était monté à la tribune pour défendre son maître. Sans lui laisser le temps de parler et de formuler sa défense, les juges lui ordonnèrent de descendre. Il fut donc tout à fait impuissant à le sauver. À l'issue de ce procès, Socrate fut ainsi condamné à boire la ciguë. De son plein gré, il refusa catégoriquement l'exil que lui offraient ses amis et ses 
disciples. Pour Socrate, l'exil était une mort lente; il préférait la mort réelle qui lui évitait la déchéance de la vieillesse. «Socrate serait mort, dira Platon, pour sauver la philosophie».

\section{Désertion et mystérieuse maladie de Platon}

L'exécution de Socrate en fait eut lieu un mois après le procès. Platon n’assista pas à sa mort. Cependant, il en donne la raison au début du Phédon, dans une phrase hautement énigmatique: "Mais Platon, je crois, était malade ${ }^{55}$.» (59b) L'expression « je crois» dans la phrase et sous la plume de Platon nous laisse supposer une hésitation sur la nature de sa maladie: estce un mal physique ou un trouble de «l'âme», par exemple un malaise psychique cachant une grande douleur et une grande ambivalence? Assister son maître bien-aimé, l'accompagner dans ce moment ultime et lui dire adieu relèvent d'un devoir filial et d'un geste d'amour. Et Platon le sait très bien. Pour cela, il lui faut affronter la réalité: la mort de son maître. Dans ce moment particulier de sa vie, il ne se sent pas prêt. Il adopte plutôt par la maladie une position de déni de sa mort: par exemple en n'assistant pas à sa mort, il échappe au réel, «ce que je ne vois pas, n’existe pas». Dans ce moment historique, Platon fuit cette vérité trop souffrante; il ne peut et ne veut le quitter maintenant. Il lui faudra en effet beaucoup de temps; il remettra la représentation de cette scène à l'ordre du jour 15 ou 17 ans plus tard quand il écrira le Phédon.

La période d'inactivité et de retrait à Mégare près d'Euclide et Terpsion, deux disciples de Socrate, a duré un temps difficile à évaluer avec certitude; elle s'étend entre 399 av. J.-C., date de la mort de Socrate et 395, celle de la guerre de Corinthe à laquelle il participa avec ses deux frères, Glaucon et Adimante. C'est aussi durant cette même période intermédiaire qu'il commence à écrire ses premiers dialogues, dits socratiques. Ne serait-ce pas là ses premiers pas de sortie de cette période dépressive? Il y a en effet dans ceux-ci une forte tendance apologétique: il veut rétablir dans l'esprit des Athéniens la réputation entachée de son maître. De fait, dans les années qui suivirent sa mort, des pamphlets contre Socrate (par ex., ceux de Polycrates) continuèrent d'être publiés à Athènes. Il est probable aussi que Platon, devant cette perte énorme, se devait d'accomplir un travail de deuil immense, si on en juge par l'importance qu'il donne à Socrate dans la plupart de ses dialogues; seulement dans trois de ceux-ci sur trente-cinq, Socrate est absent. 


\section{Sens et raisons du choix d'écriture sous la forme de dialogue}

À quels besoins particuliers répondait chez Platon cette forme si spéciale d'écriture? Celle-ci d'ailleurs n'était pas nouvelle, plusieurs disciples de Socrate l'auraient utilisée. Selon V. Goldschmidt, qui nous a laissé une grande étude sur les dialogues de Platon ${ }^{56}$, elle aurait été choisie précisément parce que le dialogue répondait parfaitement au souci de Platon de mettre l'élève ou le lecteur dans une disposition intérieure de recherche; donc, il y avait là un but purement pédagogique. «Le vrai éducateur disait Nietzsche, pose les questions, mais il n'y répond pas.»

Parmi les nombreux commentateurs, A. Thibaudet, dans son remarquable ouvrage sur Socrate ${ }^{57}$, insiste à juste titre sur son héroïsation. C'est un aspect évident qui prédomine dans chaque dialogue où il est présent : «Ainsi au Socrate condamné par les héliastes succède, dans les Champs-Élysées de la mémoire, le Socrate héroïsé pour toujours par l'art de Platon. »

L'utilisation de cette forme d'écriture est peut-être aussi un choix inconscient. Devant la souffrance ${ }^{58}$, Platon est contraint de réfléchir, de chercher une façon d'exprimer et construire avec elle, et enfin de tempérer son indignation que l'on sent encore présente dans la Lettre VII, écrite en 354 av. J.C. à l'occasion de l'assassinat de Dion, soit sept ans avant sa propre mort en 347. Cette activité d'écriture commencée après la disparition de Socrate ne s'est jamais arrêtée. À ce sujet, j'ai avancé l'hypothèse de la valeur antidépressive et thérapeutique qu'a pu présenter pour Platon cette expérience créatrice. Tous les personnages sont choisis par lui si judicieusement, et mis en scène et en discussion avec Socrate à la façon d'un rêve éveillé ${ }^{59}$. C'est Platon, je me permets de nouveau d'y insister, qui tient la plume et les fait revivre pour nous (au moment de la rédaction, ils sont tous morts); il les fait réagir et parler entre eux et Socrate dans des petites scènes bien dramatisées. "Voyez, semble-t-il nous dire, mon maître, le plus juste et le meilleur d'entre les hommes n'est pas mort, mais il est toujours vivant et il le sera toujours. » Il poursuit là une intention inconsciente de le rendre immortel. Il le montre vivant à la façon du jeu du «for da » du petit-fils de Freud, que celui-ci observait en train de jouer durant l'absence prolongée de sa mère: « tu es partie là (for, loin), je te fais revenir là (da, voilà). Magiquement, l'absence se convertit en présence; par le jeu, imaginairement, l'enfant maîtrise l'absence et la présence de sa mère ${ }^{60}$.»

Par son œuvre immense, Platon a réalisé ainsi son désir de rendre Socrate immortel, si on considère le nombre considérable d'écrits répertoriés - environ 2450 volumes et articles - concernant Socrate ${ }^{61}$. L'étude du personnage 
apparaît inépuisable. Les recherches sur Socrate auront accompagné certains auteurs, tels Thibaudet, Vlastos, Hadot, etc., durant toute leur vie de professeur et d'écrivain. Chaque écrit relève un aspect particulier de Socrate, parfois inédit, mais chacun y met aussi du sien.

À travers ses nombreux dialogues, Platon vise, semble-t-il, à nous montrer ce que Socrate lui a d'abord appris, et, de plus en plus, il ajoute ses propres idées, idées qui viendront contredire celles de son maître. Alors, peut-on penser au meurtre imaginaire du père Socrate comme il l'a avancé à l'égard de Parménide dans le Sophiste? Cela n'est-il pas inévitable? Ce désir inconscient fait partie de la trajectoire de tout disciple qui se développe et devient lui-même; Platon le note aussi au sujet d'Aristote, quand il dit que «celui-ci l'avait frappé du talon comme un poulain qui donne une ruade à sa mère ${ }^{62}$ ». Il est vrai aussi qu'Aristote a beaucoup critiqué et même renversé la « théorie des idées» de Platon.

Pourtant, malgré la lecture répétée de son œuvre, il reste très difficile de départager ce qui appartient à l'un et à l'autre. G. Vlastos s'y est appliqué dans un travail long et minutieux qui fait référence à cette question. L. A. Dorion, dans son livre très précis sur Socrate, complète remarquablement le travail de Vlastos ${ }^{63}$. Platon ne s'exprime jamais à la première personne -à l'exception d'une fois-mais se cache plutôt derrière les personnages qu'il met en scène et fait débattre devant nos yeux ses propres recherches et même son évolution personnelle: comment il devient philosophe. Sa naissance comme philosophe se serait-elle faite à partir de la résolution de son deuil, à la suite de la perte de son maître? Est-il vraiment sorti de cette grande perte réelle? Il est difficile de l'affirmer. Cependant, un doute persiste quand on relit la Lettre VII dans laquelle il exprime encore ouvertement son indignation (aganaktein). Probablement, Platon a accompli sur lui-même un travail psychique important qui lui a permis d'accéder à une certaine maturité comme homme et comme philosophe. Et un long voyage d'exil à l'étranger de 390 à 387 av. J.-C. va intensifier cette évolution ; on le retrouve ainsi en Égypte, en Cyrénaïque (Libye), en Grande-Grèce, où il rencontra les pythagoriciens et enfin à Syracuse en Sicile. À Tarente, il fit la connaissance d'Archytas, philosophe pythagoricien, mathématicien, homme d'État avec lequel il noua une longue amitié et peut-être fut-il aussi pour Platon un second maître. J. F. Mattéi nous rappelle l'importance et le sens de ce voyage dans l'évolution de Platon: «Si Socrate ne quitta jamais la caverne, comme il ne quitta jamais sa patrie, c'est Platon qui fit le premier l'épreuve de l'étranger en s'arrachant au monde natal pour en éprouver le sens ${ }^{64}$.» 
À son retour de voyage, en 387, après un long moment de transition, nous retrouvons Platon dans une étape particulière de sa vie: il a des projets d'enseignement et il fonde l'Académie qui devient la première école de philosophie à Athènes ${ }^{65}$. La philosophie connaîtra alors une grande évolution: elle acquerra l'état d'une véritable discipline qui embrasse l'ensemble de la personne: cette école offrira à chaque étudiant un mode de vie. Est-ce une influence pythagorienne? Probablement, mais elle est aussi en continuité avec ce qu'il a reçu de Socrate. De plus, ne nous fait-il pas la démonstration, par sa propre démarche intérieure, du long chemin que chaque disciple devra traverser pour devenir philosophe, voire un modèle de transmission? Enfin, sur ce plan, n'y a-t-il pas un parallèle à établir avec la naissance de la psychanalyse et celle de Freud comme psychanalyste? C'est ce que je vais essayer de dégager en m'inspirant d'un des plus beaux dialogues de Platon: le Banquet, écrit en 385 .

\section{Le Banquet et I'évolution de Platon}

Il ne faut jamais oublier - je le rappelle une autre fois avant d'entrer dans ce dialogue - que les propos tenus par les personnages sont créés par l'auteur, Platon qui tient la plume et élabore tous les discours. Il leur fait exprimer ce que lui, Platon, sait de l'amour. Le Banquet est une «joute érotique» selon l'expression juste de Thierry Ménissier ${ }^{66}$, que nous expose Platon. Il nous livre en effet un Platon en train de progresser et de chercher une voie sublimatoire de ses pulsions amoureuses et sexuelles. Platon est-il pédéraste ou homosexuel? De toute façon, on lui reconnait plusieurs conquêtes: Aster ou Phèdre, Dion de Syracuse, Alexis (?) et même Agathon chez lequel a lieu le fameux Banquet. Il n'aurait connu qu'une seule femme, du nom d'Archéanassa, courtisane de Colophon ${ }^{67}$ dont on ne sait que peu de chose, sinon son nom ${ }^{68}$.

Il est frappant de constater, comme le relève Ménissier, «la permanence du thème de l'homosexualité» dans les dialogues. Dans le Banquet, Aristodème serait un des amoureux les plus passionnés de Socrate; dans le Protagoras, il est dit qu'Agathon et Pausanias sont amants; dans Euthydème, Ctésippe serait l'éraste de Clinias; dans Ménon, Aristide, celui de Ménon; dans Parménide, Zénon aurait été le «mignon » (païdika) de Parménide ${ }^{69}$.

D’abord, il faut rappeler que la pédérastie (païdérastia) n’avait pas le même sens que celui que l'on attribue aujourd'hui au terme de pédophilie. Il n'y a pas de mot dans le vocabulaire grec ancien pour traduire ce sens. La pédérastie (paidos érastes) qui veux dire amour des garçons, avait un rôle 
important dans l'éducation et la formation des jeunes Grecs. Ceci venait d'une longue tradition militaire; le couple aimant-aimé (érastes et éroménos) et guerrier était considéré comme redoutable dans la bataille. L'idéal guerrier a toujours existé en Grèce ancienne ; un garçon devient homme lorsqu'il est guerrier et une fille devient femme par le mariage ${ }^{70}$. Pour Socrate et Platon, cette importance de la pédérastie est souvent rappelée et elle est nommément au cour du Banquet comme nous allons le voir. De fait, ils s'appuient tous les deux sur une longue tradition antique.

\section{Les intentions de Platon}

Que fait Platon dans le Banquet? Se permet-il d'exposer les conceptions de l'amour qui ont chronologiquement précédé celle qu'il nous exposera à la fin du Banquet dans le discours de Socrate-Diotime? Il nous donne à première vue l'impression de vouloir régler ses comptes avec les poètes, en l'occurrence ici: Phèdre, Agathon, et surtout Aristophane qu'il fait «hoqueter» juste au moment où il doit prendre la parole. On sait combien ce dernier a ridiculisé Socrate en le raillant dans sa pièce Les Nuées. Petite vengeance? La haine et l'envie sont d'ailleurs évidentes à travers son œuvre. Auguste Dies ${ }^{71}$, dans son introduction si remarquable de La République, le note et en vient à la conclusion qu'il existe chez Platon une intention non avouée d'occuper la première place: «il n'y a pas de meilleur que moi», donc pas de rival possible.

Ou encore, nous communique-t-il sa propre évolution, celle qui est en mouvement dans ce dialogue? En ce sens, Platon, par ses personnages, nous révèlerait ses conceptions anciennes, et il tenterait ici de les dépasser sous nos yeux en en exposant une nouvelle, dans le discours de Diotime, à laquelle Socrate (ou Platon) a donné la parole. Diotime nous trace aussi à la fin de son discours les voies ou les étapes de progression (sublimatoire) de cette métamorphose d'Éros: comment Éros devient éducateur et philosophe.

Pour Socrate (et Platon), "philosopher», c'est "amener une belle âme à la réalisation d'elle-même ${ }^{72}$ ", la féconder et la faire éclore (accouchement). Écoutons Socrate: «C'est là tout ce que mon art peut faire, et rien de plus. Je ne sais rien de ce que savent les grands et admirables sages de ce temps et du temps passé. Quant à l'art d'accoucher, ma mère et moi, nous l'avons reçu de Dieu, elle pour les femmes, et moi pour les jeunes gens d'âme généreuse et pour tous ceux qui sont beaux ${ }^{73}$."

Afin d'accomplir cette œuvre, Socrate ira "chasser» les jeunes gens au gymnase comme Platon le montre dans Charmide. Tel est le sens de la 
philosophie socratique: « cette chasse sera pédérastique et philosophique ${ }^{74}$.» Dans le Gorgias, Socrate affirme à Calliclès que la philosophie est son «pä̈dika» (son mignon), son garçon préféré.

\section{Passage de la pédérastie à la philosophie}

Pour cerner ce moment dans le Banquet, il est nécessaire de nous centrer sur le discours de Socrate. Dans celui-ci, il nous rapporte ses entretiens de jadis avec Diotime, prêtresse de Mantinée, entretiens probablement fictifs comme l'est le personnage de Diotime ${ }^{75}$. Dans ce discours, Socrate raconte (ou Platon imagine) comment s'est passée son initiation à la philosophie. Mais en dernière instance et sous-entendu, Platon ne nous parle-t-il pas de sa propre initiation avec Socrate? Et de plus, pourquoi confie-t-il ce rôle à une femme, de surcroît, étrangère et prêtresse? Plus loin, je tenterai de répondre à cette question.

Dans une première partie $(201 \mathrm{~d})^{76}$, Socrate nous dit qu'il a utilisé avec Agathon le même langage que Diotime avec lui, et la même méthode d'interrogation et de réfutation, c'est-à-dire la maïeutique. Peut-on supposer qu'il a appris cette méthode de Diotime qui réfutait son opinion sur l'amour, à savoir que l'amour, contrairement à ce qu'il pensait n'était ni beau, ni bon, ni laid, ni mauvais? (201e) Je me limiterai à résumer les propos de Diotime et à énumérer les questions importantes soulevées par sa dialectique, pour me concentrer sur la fin de son discours qui a trait à «l'initiation à la philosophie».

\section{Quelle est la nature d'Éros?}

Cette interrogation de Diotime nous conduit à découvrir que l'amour est un intermédiaire entre le beau, le bon, et le laid, le mauvais, de même que entre la science et l'ignorance, et un intermédiaire entre le mortel et l'immortel (202 a b c). Tout intermédiaire est démonique. Tout ce qui est démonique rend possible aux dieux, dit Diotime, de communiquer avec les hommes et de les entretenir pendant la veille et le sommeil (203a). Diotime est dite prêtresse, donc aussi un personnage démonique. On pense ici à la voix intérieure dont nous parle Socrate comme un démon. « Il y a une grande variété de démons. Parmi eux, il y a l'amour» (203 a).

\section{Origine d'Éros: sa naissance et sa condition singulière}

Il est le fils d'Expédient (Poros), son père et de Pauvreté (Pénia), sa mère. Celle-ci, désirant un enfant de Poros, s'étendit près de lui endormi. Et elle 
devient grosse d'Éros (203 b, c). Cette double origine divine (Poros veut dire aussi passage, donc le dieu « du passage ${ }^{77}$ ») et mortelle détermine sa condition : comme sa mère, il est toujours pauvre, misérable, rude, malpropre, et n'est ni beau, ni délicat. Mais il tient aussi de son père, Poros : il est à l'affût de tout ce qui est beau et bon (ce qui lui manque toujours), incomparable sorcier, magicien d'idées et d'expédients, passant sa vie à philosopher; ainsi il serait toujours en train de se sortir de la misère transmise par sa mère. Il n'est ni mortel ni immortel (203 b c d e), mais intermédiaire entre les deux.

\section{Les bienfaits d'Éros}

«Éros est à mi-chemin entre le savoir et l'ignorance». S'occuper de philosophie et acquérir le savoir n'est pas le propre des dieux, puisque le savoir, ils le possèdent déjà, et que les ignorants ne sont pas enclins à acquérir le savoir. "Ce sont les intermédiaires entre les deux espèces qui s'occupent de philosopher, et Éros est l'un d'eux. De par son origine, il doit nécessairement tendre vers le savoir et tenir le milieu entre celui qui sait et l'ignorant» (204a b). En résumé, Éros est l'amour des belles choses (du Beau), plus précisément le désir et la possession du Beau et cette possession rend heureux. N'est-ce pas là pour Platon la définition du bonheur ${ }^{78}$ ?

Cette inquiétude et cette aspiration de l'Amour d'avoir à soi toujours ce qui est bon et beau et de le posséder, où conduisent-elles les êtres mortels que nous sommes? La réponse de Diotime est la suivante: vers «le désir d'immortalité ». Ils cherchent à l'accomplir dans l'union, «la procréation et dans l'accouchement dans de belles conditions ». « Pour un être mortel, la génération équivaut à la perpétuation dans l'existence, c'est-à-dire à l'immortalité » (206 e).

L'immortalité devient pour les humains l'objet de leur amour. «C'est plutôt, $j$ 'imagine, dit Diotime, pour que leur excellence reste immortelle et pour obtenir une telle renommée glorieuse que les humains dans leur ensemble font tout ce qu'ils font...» (208 c) Cependant en tant qu'hommes, nous n'atteindrons que des substituts d'immortalité. «Ceux qui sont féconds selon le corps se tournent de préférence vers les femmes; et leur façon d'être amoureux, c'est de chercher en engendrant des enfants, à s'assurer, s'imaginentils, l'immortalité, le souvenir et le bonheur, pour la totalité du temps à venir » (208 e). «Et il y a ceux qui sont plus féconds dans leur âme que dans leur corps. » Diotime (Platon) aborde ici le problème de la création. Ceux-ci cherchent à «s'assurer ce dont la gestation et l'accouchement reviennent à l'âme » et cela par l'excellence de la pensée et la recherche de la beauté. Elle nomme 
les poètes et les artistes, "procréateurs et inventeurs », ceux qui possèdent «la partie la plus haute et la plus belle de la pensée; celle qui concerne l'ordonnance des cités et des domaines» (209 a).

\section{L'amour éducateur}

Diotime (Platon) va nous introduire maintenant dans le sujet qui est le centre de ma réflexion à savoir l'initiation proprement dite du philosophe, sur le passage de la pédérastie à la philosophie, ascension vers les formes intelligibles, le beau en soi.

Or parmi ces hommes maintenant, «il s'en trouve un qui est fécond selon l'âme depuis son jeune âge, parce qu'il est divin, et que, l'âge venu, il sent le désir d'engendrer et de procréer, bien entendu cherche, j'imagine, en jetant les yeux de tous les côtés, la belle occasion pour procréer; jamais, en effet, il ne voudra procréer dans la laideur. Aussi s'attache-t-il, en tant qu'il est gros, aux beaux corps plutôt qu'aux laids, et s'il tombe sur une âme qui est belle, noble et bien née, il s'attache très fortement à l'une ou l'autre de ces beautés, et, devant un individu de cette sorte, il sait sur-le-champ parler avec aisance de la vertu, c'est-à-dire des devoirs et des occupations de l'homme de bien, et il entreprend de faire l'éducation du jeune homme» (209 b c).

Dans cette partie du discours de Diotime, que j'ai ici cité intégralement, Platon nous parle de sa conception de l'éducation et la formation du philosophe, celle que Socrate lui a transmise, mais aussi celle que lui-même est en train d'élaborer et de mettre en place à l'Académie fondée en 387 et où il enseignera jusqu'à sa mort en 348. Et de plus, il nous révèle l'état d'esprit et de passion qui l'anime. Écoutons encore Diotime le préciser : «C'est que, j’imagine, au contact avec le bel objet et dans une présence assidue auprès de lui, il enfante et procrée ce qu'il portait en lui depuis longtemps; qu'il soit présent ou qu'il soit absent, sa pensée revient à lui, il nourrit ce qu'il a procréé. Ainsi une communion bien plus intense que celle qui consiste à avoir des enfants, une affection bien plus solide s'établissent entre de tels hommes; plus beaux en effet et plus assurés de l'immortalité sont les enfants qu'ils ont en commun» (209 c). Diotime cite en exemple Homère, Hésiode et les autres grands poètes qui ont laissé « des poèmes immortels », d'autres comme Lycurgue du côté de Sparte et Solon du côté d'Athènes, honorés comme les pères des lois, tous ont été par leurs créations les grands éducateurs de la Grèce ${ }^{79}$. Platon envie la gloire de ces grands hommes et se voudrait leur continuité. Qui ne le souhaiterait pas s'il n'était comme Platon, un fils de descendants d'ancêtres si glorieux tels Solon et Codros, en plus d'être amoureux d'Homère et Pindare? 
Toutes ces figures citées ici, nous pensons surtout à Solon et Lycurgue, représentent pour lui des modèles idéaux d'identification qui l'accompagneront dans l'élaboration de ses deux grandes ouvres: La République et Les Lois.

À ce point de son discours, Diotime énumère à Socrate les cinq étapes de l'initiation proprement dite de l'ascension vers le beau: au premier degré, «s'il est bien dirigé par celui qui le dirige, il n'aimera qu'un seul corps »; au deuxième degré, «il saisit que les beaux corps sont le reflet d'une même beauté corporelle, passant d'un beau corps à deux et à tous les corps». "Il deviendra amoureux de tous les corps et son impérieux amour pour un seul être se relâchera, il le dédaignera» (210 b). L’attachement ne sera pas entièrement défini par l'attraction corporelle; au troisième degré, « en regardant la beauté dans les âmes, il saisira que cette beauté est de plus grande valeur que la beauté des corps » $(210 \mathrm{~b})$; au quatrième degré, «ayant perçu que l'esprit est source de beauté, il éprouvera la beauté de la vie dans la cité et des lois qui la gouvernent», et il est forcé « de constater que cette beauté est toujours semblable à elle-même ». Maintenant, c'est vers les sciences que le guide dirigera son élève.

Comment comprendre ce que Diotime (Platon) veut nous communiquer? Il est clair que l'élève doit abandonner cet investissement premier trop fusionnel. C'est la sortie de la "caverne du même» où le guide et l'élève risquent l'emprisonnement à l'intérieur. Diotime cite d'ailleurs trois exemples d'enfermement qui menacent l'élève (210d). Entraîné par le mouvement d'Éros qui est aussi un conquérant, l'élève, avec le support du guide, est contraint d'agrandir son champ d'investissement; d'un beau corps, son regard se porte ailleurs vers la lumière, d'abord vers "d'autres beaux corps », ensuite il découvre la grandeur de la beauté de l'âme, des sciences, le savoir et la connaissance, la recherche de la vérité et l'importance de la pensée (phronésis) pour enfin parvenir à une cinquième et ultime étape: la connaissance unique qui est la beauté en soi, transcendante, absolue et éternelle. "Toutes les fois donc, nous dit Diotime, que, en partant des choses d'ici-bas, on arrive à s'élever par une pratique correcte de l'amour des jeunes garçons, on commence à contempler cette beauté-là, on n'est pas loin de toucher au but. Voilà donc quelle est la droite voie qu'il faut suivre dans le domaine des choses de l'amour ou sur laquelle il faut se laisser conduire par un autre...» (211 b)

Ici, on est véritablement dans les premiers temps de la formulation de la philosophie platonicienne; elle sera remaniée bientôt dans le Ménon, le Sophiste, le Parménide, Timée. Dans le Banquet, il est parvenu à une étape importante de son évolution intérieure. L'initiation, qu'il formule et qu'il met 
dans la bouche de Diotime, n'est-elle pas la sienne en ce moment crucial où il se détache de son maître, Socrate, en 384, c'est-à-dire 15 ans après la mort de celuici? Dans le Phèdre, il complétera l'exposition de sa conception de l'amour éducateur.

\section{La sublimation}

Arrêtons-nous un moment sur cette question de la sublimation telle qu'elle fut définie par Freud et reprise par Laplanche et Pontalis. Cette définition pourrait s'appliquer ici parfaitement: «La pulsion sexuelle met à la disposition du travail culturel des quantités de force extraordinairement grandes, et ceci, par suite de cette particularité, spécialement marquée chez elle, de pouvoir déplacer son but sans perdre, pour l'essentiel, son intensité. On nomme cette capacité d'échanger le but sexuel originaire contre un autre but, qui n'est pas sexuel, mais qui lui est psychiquement apparenté, capacité de sublimation ${ }^{80}$.»

Dans l'état actuel de la recherche en psychanalyse, la capacité sublimatoire s'exerce durant toute la cure avec des moments oscillatoires, où l'amour de transfert se déplace graduellement de l'analyste vers le processus lui-même; une élaboration, une théorisation plus intensive se fait jour et en même temps une séparation s'amorce, un deuil s'installe lentement, et graduellement l'analysé prendra en main son analyse. Celle-ci deviendra désormais une auto-analyse ${ }^{81}$ qu'il pourra continuer après la terminaison des séances. Ceci est plus évident et fondamental quand il devient lui-même analyste. À cet égard, on peut se demander s'il n'en fut pas ainsi en philosophie antique, du moins chez Platon: l'amour pour le maître se déplaçant et se concentrant sur la recherche philosophique, celle qui désormais prévaudra pour chaque élève. Ceci me paraît évident dans le discours de Diotime, précisément dans l'initiation formulée par elle (Platon).

\section{La métaphore de la procréation et de l'accouchement et le processus de création}

Depuis l'antiquité, on le constate ici dans la pensée de Socrate et de Platon, l'acte créateur se conçoit en se référant à la fécondation, à la procréation et à l'accouchement ${ }^{82}$; ceux-ci servant de métaphores. N'en est-il pas ainsi encore aujourd'hui? Et n'y a-t-il pas là pour Platon, entre autres, une raison du choix d'une femme pour exposer sa conception de la transmission de la philosophie? La femme, par son intériorité particulière, ses qualités maternelles: elle peut donner la vie et généralement ses capacités 
régressives seraient plus facilement mobilisables que celles de l'homme, pour rejoindre le nouveau-né. Elle serait plus en mesure de comprendre et d'expliquer en quoi consiste une pratique correcte de la pédérastie. De plus, en donnant la parole à une femme, Platon fait parler son féminin en lui, ceci est un point très important ${ }^{83}$. Est-il besoin de rappeler que celui-ci n'est pas le propre de la femme comme le masculin d'ailleurs n'est pas le propre de l'homme? Grâce à ses identifications primaires, chacun est bisexuel psychiquement.

La création met en jeu ce féminin. Goethe nous a parlé de l’Éternel Féminin, le monde des Mères. «Les Mères! Les Mères! Des mères! - cela résonne si étrangement ${ }^{84}$ !» Forme ou Savoir insaisissable dont Faust attend la clef qu'il espère obtenir de Méphisto. Rilke parlera de son côté de "l'Ouvert ${ }^{85}$ », une sorte de "Nulle part sans nom» absolu qui ouvre sur un monde inconnu, «terra incognita», de l'inquiétante étrangeté du savoir de l'inconscient que la création nous fait toucher du doigt. Pour Freud, la clef des Mères est l'ouverture de l'inconscient et la quête de l'origine. Cette clef qu'il attendait de Breuer, ensuite de Fliess, il la trouvera dans l'analyse de ses propres rêves, l'une des plus importantes voies d'accès à l'inconscient. N'est-ce pas cet absolu que nous indique Platon dans la contemplation de la Beauté, comme Principe premier de toute connaissance?

La prise de conscience de courant féminin donc homosexuel que Freud appelle «androphile», est un moment fondamental et créateur dans toute analyse. L'acceptation, l'écoute réceptive et bienveillante de l'analyste et la perlaboration de ce transfert positif, c'est-à-dire la mise en représentation de tout ce qui le concerne, marque de façon définitive l'analyse et l'avenir de l'analysé. En effet, en acceptant cet amour de transfert, en le perlaborant, il pourra désormais s'en détacher et prendre en main son destin; la relation peut s'interrompre à une date fixée par les deux. Dorénavant, il pourra continuer seul son analyse.

Comme Freud l'a précisé, "l'amour de transfert ${ }^{86}$ " n'est pas l'apanage de la cure psychanalytique, quoique dans celle-ci, tout est en place pour qu'il se déploie de façon plus évidente. Mais on peut le rencontrer dans plusieurs situations relationnelles, en particulier dans la relation maitre-élève. Dès sa première rencontre avec Socrate, et aussi à partir de la lecture de ses dialogues, on peut facilement conclure qu'il en fut ainsi pour Platon et de même pour son célèbre disciple, Aristote. Pour l'homme grec en général, la reconnaissance de ce mouvement féminin dans la relation affective ne devait pas être trop visible. N'en serait-il pas de même pour Platon? «Platon nous cache ce qu'il 
pense tout autant qu'il nous le révèle », nous dit Alexandre Kojève ${ }^{87}$. Le dernier discours du Banquet, celui d'Alcibiade, nous montre bien l'intensité de son attachement à condition que l'on ne dénie pas que c'est Platon lui-même qui nous introduit dans cette relation ${ }^{88}$.

Lacan, d'ailleurs, dans une longue réflexion sur le transfert, s'est servi de ce dialogue comme d'un exemple clinique pour énoncer et construire sa propre théorie. Il utilise ce que Platon montre dans ce dialogue, mais en ajoutant une interprétation analytique qui me paraît assez juste mais incomplète; en effet, dans son interprétation, il n'établit pas le lien avec la situation infantile pourtant bien indiquée par Platon ${ }^{89}$. Il est étonnant aussi que ce discours d'Alcibiade soit passé sous silence par plusieurs commentateurs qui n'y ont vu qu'une autre apologie de Socrate.

Je résumerai brièvement la trajectoire de ce long discours d'Alcibiade. J'insisterai sur les moments importants de celui-ci pour élaborer ma compréhension. Tout d'abord, Alcibiade se présente comme un coup de tonnerre; ivre, tonitruant, subversif même, comme Éros, il fait ainsi son entrée chez Agathon où a lieu le banquet. Alors que les invités ont pris le parti de ne pas trop boire et de faire l'éloge de l'amour, chacun à tour de rôle, on lui rappelle cette entente. En revanche, Alcibiade propose de boire sans retenue et de faire l'éloge non pas de l'amour, mais de Socrate, plus précisément, il parlera de sa relation amoureuse avec Socrate. Au début, dès l'apparition d'Alcibiade, Socrate fait deux mises au point qui visent à éviter les débordements. 1) Il demande à Agathon, l'hôte, d'être vigilant; il avoue qu'il a eu une relation amoureuse avec Alcibiade et ce dernier s'est montré jaloux. 2) Quand Alcibiade annonce qu'il va faire l'éloge de Socrate avec ces mots: «je dois m'attaquer à ce personnage et lui infliger la punition qu'il mérite devant vous ", Socrate intervient: "Qu'as-tu en tête? Tu vas faire mon éloge en faisant rire à mes dépens?» Alcibiade répond: "Je dirai la vérité; à toi de voir si tu me le permets. » Socrate répond: «La vérité, mais bien sûr je te permets de la dire; c'est même ce que je t'ordonne de faire.» (214 e) Voilà, la règle est prononcée et le rôle du maître, affirmé.

Pour esquisser le portrait de Socrate, Alcibiade utilise deux images très fortes. 1) Socrate est pareil à ces silènes que l'on présente dans les ateliers de sculpteurs. Ils sont représentés avec un aulos à la main. Si on les ouvre au milieu, on aperçoit, à l'intérieur, des figurines des dieux. 2) Socrate ressemble aussi au Satyre Marsyas, physiquement d'abord (il était laid comme Socrate), mais pas seulement, il n'est pas comme lui un joueur d'aulos dont les airs sont capables « de nous mettre en état de possession ». Socrate n'est pas 
un joueur d'aulos, «c'est en proférant de simples paroles qu'il produit le même effet » (215 e). En somme, Socrate est un silène parce qu'il possède à l'intérieur de lui des trésors divins (agalma) qui excitent le désir d'Alcibiade. De plus, il est aussi une sorte de satyre comme Marsyas; par ses discours, il peut nous troubler et nous posséder : «mon cœur, dit Alcibiade, bat plus fort que celui des Corybantes, ses paroles me tirent des larmes...» «Mais lui ce Marsyas, il m'a bien souvent mis dans un état tel qu'il me paraissait impossible de vivre comme je le fais...» Même s'il ne rencontre Socrate que très rarement, cette image idéalisée est encore intacte et bien présente: «encore maintenant, dit-il, si j'acceptais de lui prêter l'oreille, je ne pourrais pas rester insensible et j'éprouverais les mêmes émotions» (216 a).

Aux prises avec l'envahissement intérieur que lui faisait ressentir sa relation amoureuse, Alcibiade ne voyait pas d'autre choix que de tenter de fuir en se lançant dans la politique: "Je me fais violence, je me bouche les oreilles, comme pour échapper aux Sirènes. Je m'éloigne en fuyant pour éviter de rester assis là à attendre la vieillesse auprès de lui. » Il lui arrive même de souhaiter la mort de Socrate: «Souvent, j'aurais plaisir à le voir disparaître du nombre des hommes. » Mais, il ne veut pas le perdre non plus : «Si cela arrivait, je serais plus malheureux encore » (216 c).

Poursuivant sa comparaison avec le Silène, Alcibiade veut nous convaincre qu'il a raison de s'enfuir. De quelle nature est cette emprise de Socrate sur lui ? «Il a un penchant amoureux pour les beaux garçons et il est troublé par eux. D'un autre côté, il ignore tout et dit qu'il ne sait rien, c'est du moins l'air qu'il se donne» (Il s'agit ici de l'ironie socratique). Ce n'est pas la beauté physique et la richesse du garçon qui l'intéresse. «Pour lui, tous ces biens n'ont aucune valeur, et nous ne sommes rien à ses yeux...» (216 e) Écoutons attentivement ici la suite du discours d'Alcibiade où il nous montre son désir pour Socrate: "Mais, quand il est sérieux et que le silène s'ouvre, je sais si quelqu'un a vu les figurines qu'il recèle. Moi, il m’est arrivé de les voir et elles m'ont paru si divines, si précieuses, si parfaitement belles, si extraordinaires que je n'avais plus qu'à exécuter sans retard ce que me recommandait Socrate» (217 a).

Alcibiade nous expose la force qui l'attirait vers Socrate et l'envahit totalement: il a vu ce qu'il lui manque et que Socrate possède, du moins il en est persuadé; et il a le désir brûlant de l'avoir. Il a pensé que Socrate était amoureux de lui, maintenant c'est lui qui est épris de Socrate. Pour s'en sortir, il a le projet de le séduire et ainsi de le dominer et de cette façon, obtenir de Socrate ce qu'il a vu en lui et qu'il désire. 
Parce qu'il croyait que Socrate était toujours amoureux de lui, par deux fois, il tenta de l'attirer dans ses filets. Une première fois, Socrate s'esquive et lui échappe. La seconde fois, il prolonge le repas et les discussions très tard dans la nuit et l'oblige à rester. Que s'est-il vraiment passé? Alcibiade doit tout dire, comme Socrate lui a ordonné au début. Mais, il hésite, et il a peur de ne pas être compris: «Mon état est aussi celui d'un homme qu'une vipère mâle a mordu... » La morsure est profonde et douloureuse, «car, c'est au cœur ou à l'âme que j'ai été frappé et mordu par les discours de la philosophie lesquels blessent plus sauvagement que la vipère quand ils s'emparent d'une âme jeune qui n'est pas dépourvue de talent, et qu'ils lui font commettre et dire n'importe quelle extravagance» (218 a). Personne d'autre que ceux qui ont connu le même état peuvent le comprendre ${ }^{90}$. C'est pourquoi, il demande le support et la compréhension des autres invités pour poursuivre son récit.

Alcibiade raconte tout avec une grande franchise. Cependant, il se place ${ }^{91}$ dans la position d'un amant (erastes) qui parlerait à son amoureux (eromenos). «Je pense, repris-je, que tu es un amant digne de moi, le seul qui le soit, et je vois bien que tu hésites à m'en parler. En ce qui me concerne, voici ce qui en est. J'estime qu'il est tout à fait déraisonnable de ne pas céder à tes vœux sur ce point aussi comme toute autre occasion où tu aurais besoin de ma fortune ou de mes amis. Rien à mes yeux ne présente plus d'importance que de devenir le meilleur possible, et j'imagine que, dans cette voie, je ne puis trouver maître qui soit mieux en mesure de m'aider que toi. Dès lors, devant ceux qui savent à quoi s'en tenir, je serais beaucoup plus honteux de ne point céder aux vœux d'un homme comme toi, que je ne le serais, devant le grand nombre qui ne sait pas à quoi s'en tenir, de céder à ses vœux» (218 d).

Socrate va intervenir en le rappelant de nouveau à l'ordre: «Mon cher Alcibiade, il y a des chances pour qu'en réalité, tu ne sois pas si maladroit, à supposer toutefois que ce que tu dis sur mon compte est vrai et que j'ai le pouvoir de te rendre meilleur. Tu vois sans doute en moi une beauté inimaginable et bien différente de la grâce que revêt ton aspect physique. Si donc, l'ayant aperçue, tu entreprends de la partager avec moi et d'échanger beauté contre beauté, le projet que tu comptes faire à mes dépens n'est pas mince; à la place de l'apparence de la beauté, c'est la beauté véritable que tu entreprends d'acquérir, et en réalité, tu as l'idée de troquer l'or contre du cuivre. Mais, bienheureux ami, fais bien attention, de peur tu n'ailles t'illusionner sur mon compte, car je suis rien» (218 e 219 a).

Alcibiade rassure Socrate qu'il a dit exactement tout ce qu'il pensait et s'abandonne à son jugement. Après avoir étendu sur lui son manteau, il 
s'allonge sur le sien en enlaçant de ses bras «cet être véritablement divin et extraordinaire et rest(e) couché contre lui toute la nuit» $(219 \mathrm{~b}, \mathrm{c})$.

Par son interprétation, Socrate l'avait ramené judicieusement à la relation maître-élève, sous-jacente à celle de père-fils, comme le dit d'ailleurs Alcibiade à la fin de son récit: «je me levai après avoir dormi aux côtés de Socrate, sans que rien de plus ne se fût passé que si j'avais dormi auprès de mon père ou de mon frère aîné» ( $219 \mathrm{~d})$. Platon a bien saisi l'aspect infantile de cette relation amoureuse comme le révèle tout transfert. Celui-ci ramène le sujet à sa première relation d'amour, celle qu'il a eue avec ses parents. Il termine son discours en affirmant «qu'il s'est conduit ainsi avec beaucoup de garçons qu'il dupe en se donnant l'air d'un aimant alors qu'il tient le rôle du bien-aimé plutôt que celui de l'amant». Et il met en garde Agathon de ne pas se laisser prendre à son jeu. Socrate intervient de nouveau pour lui montrer derrière son comportement, qu'il a « déguisé subtilement ses intentions pour essayer de cacher l'objet de tous ses discours » : ton intention est "de nous brouiller, moi et Agathon, parce que tu t'imagines que c'est toi que je dois aimer et personne d'autre et parce qu'Agathon doit être aimé par toi et personne d'autre» ( $222 \mathrm{~d}$ ). Socrate a cru important de rétablir les règles qui avaient été dictées au début de ce banquet, en 177 d et rappelées aussi en 214 c. Socrate commande le respect des Lois : elles représentent la figure du père. Agathon fait une fête en l'honneur du prix qu'il vient de recevoir au concours des tragédies, et à cette occasion, il reçoit des invités. Il est l'hôte, donc le maître et c'est lui qui au début accueille Socrate et l'invite à s'étendre près de lui.

Alcibiade proteste, bien sûr, et pour cause. Son projet de l'emporter sur Socrate et de le dominer est déjoué: «Lorsque Socrate est présent à quelque part, il est impossible à un autre de tenter quelque chose du côté des beaux garçons» (223 b). Alcibiade est en colère, mais reconnaît la force, la perspicacité et l'intelligence de Socrate. Il est contraint d'admettre, pour son plus grand bien du reste, que Socrate reste le maître (le père) et lui, l'élève (le fils), dans le récit que nous en fait Platon. Le projet éducatif d'Alcibiade est loin d'être un échec. Il est plutôt remis sur les rails par un Socrate qui est perspicace et cohérent dans son rôle de maître; la formation d'Alcibiade, en toute rigueur, peut se poursuivre. Éros peut devenir philosophe. Ne l'est-il pas déjà dans la personne de Platon?

Ceci nous amène à nous poser plusieurs questions. Est-ce que ce banquet que nous présente Platon n'est pas avant tout une histoire fictive, une pièce de théâtre, qui doit être lue ainsi ? Et que ce banquet n'ait peut-être pas 
vraiment eu lieu en 416 av. J.-C., n'est-ce pas plutôt un sujet tout à fait secondaire?

Le drame d'Alcibiade a réellement eu lieu. Son histoire nous a été admirablement bien racontée par J. de Romilly ${ }^{92}$. Les conséquences graves de son évolution et de sa conduite envers son pays ont été d'ailleurs reprochées à Socrate dont le procès et la mise à mort, selon plusieurs historiens, en furent les causes sous-jacentes. Dans l'antiquité, le maître était souvent tenu responsable de la conduite de son élève. Aussi, grâce à G. Vlastov, on peut lire un fragment d'Eschine dit le Socratique ${ }^{93}$; celui-ci rapporte un commentaire intéressant de Socrate qui fait retour sur son erreur à l'endroit d'Alcibiade. En se comparant aux bacchantes qui «possédées par le dieu peuvent retirer du lait et du miel des puits...", il ajoute: "de même moi aussi, tout en ayant aucun savoir par l'enseignement duquel je puisse lui être utile, néanmoins j'ai pensé qu'en le fréquentant, je pourrais l'améliorer par mon amour. » Par son attitude, n'aurait-il pas devancé le désir de son élève? Cependant, il est tout à fait possible qu'il ait beaucoup appris de cet échec.

\section{En guise de conclusion}

Qu'est ce que Platon nous révèle avec ce long discours d'Alcibiade? Derrière le personnage d'Alcibiade, il y a évidemment Platon en 385 av. J.C. qui regarde et expose sa relation amoureuse avec Socrate, son maître, du moins ce qu'elle a été, à un moment tout à fait particulier de son histoire où il devient lui-même un maître comme Socrate duquel il se démarque, et où il reçoit maintenant des élèves à son Académie. Il a mis en place un programme d'enseignement et de recherche que chaque élève, futur philosophe, se voit proposer, et de plus un mode de vie dans lequel il choisit de s'impliquer accompagné d'un maître qui le guidera et auquel il s'attachera. Dans le discours de Diotime, il nous a parlé de cette transformation et de ce cheminement d'Éros vers les sciences et la science du beau et vers la contemplation de la beauté en soi et les étapes d'initiation pour y parvenir; c'est en somme un passage et en même temps, une méthode de transmission. Cette naissance de la philosophie et de Platon, philosophe, ressemble en plusieurs points à celle de la psychanalyse et de Freud, psychanalyste, malgré les 2400 ans qui les séparent. Dans les deux situations, nous assistons à une métamorphose d'Éros.

Pour conclure, je rappellerai une profonde réflexion avec laquelle JeanFrançois Mattéi termine son remarquable livre sur Platon ${ }^{94}$ : «Il n’y a jamais, sur le théâtre du monde, qu'une seule scène, et qu'un seul protagoniste: la 
tragédie de l'existence était déjà écrite dans la caverne avec son acteur solitaire. Nous le savons tous au fond de nous. En ouvrant un livre de philosophie, nous déroulons le palimpseste de la mémoire, et nous ravivons chaque fois les traits effacés du prisonnier de la caverne. » N'en est-il pas ainsi pour le psychanalyste chaque fois qu'il reçoit un nouveau patient en analyse? Ce dernier n'est-il pas dans la situation du prisonnier de la caverne, et l'analyste, devant l'évocation en écho de sa propre histoire? Tout comme Faust, cherchant la lumière, ce patient vient demander à Méphisto la clef des Mères qui lui ouvrira les portes de l'inconscient et le conduira à la recherche concernant ses origines ${ }^{95}$.

Jean Bossé

1530, av. Bernard O, app. 10

Outremont, Québec

$\mathrm{H} 2 \mathrm{~V} 1 \mathrm{~W} 8$

jeanbosse@videotron.ca

\section{Notes}

1. J. Bossé, 1993, D’où viennent les psychanalystes? Où vont-ils?, Trans, Politique, n 3, 2974 ; Bossé, J., 1997, Le Coq-Héron, nº 146, 3-28.

2. S. Freud, Correspondance, 1873-1939, lettre 86 à Martha, (24-11-1885), Paris, Gallimard.

3. S. Freud, 1956, La naissance de la psychanalyse, Paris, PUF, (dorénavant NP) L. 21, 29-081894, passage inédit cité in Schur, M., La mort dans la vie de Freud, Paris, Gallimard, p. 106.

4. Voir O. Mannoni, 1969, Clefs pour l'imaginaire ou l'Autre scène, Paris, Seuil, p. 125.

5. Lettre du 18-10-1893, inédite, citée in Schur, op. cit., p. 62-63.

6. Lettre 17, 19-04-1894, NP, p. 74-75.

7. Lettre 18, 21-05-1894, ibid., p. 76-85.

8. O. Mannoni, op. cit., p. 124-125.

9. L'auteur emploie les italiques pour souligner les parallèles, entre les scènes transférentielles psychanalytique (Freud-Fliess) et philosophique (Platon-Socrate).

10. Lettre inédite, 22-06 1895, in ibid., p. 115.

11. Voir la lettre du 21-05-1895, in Schur, op. cit. Ce léger vertige annoncerait un triomphe possible de Freud sur Fliess concernant l'accomplissement de ce désir.

12. Lettre inédite, 20-04-1895, ibid., p. 110.

13. D. Anzieu, 1975, L'auto-analyse de Freud, Paris, PUF, tome 1, p. 200-204.

14. M. Schneider, Les blessures de mémoire, Paris, Gallimard.

15. NP, p. 307-396.

16. O. Mannoni, op. cit., p. 129.

17. Lettre 65, 12-06-1897, NP, p. 187.

18. Lettre 66, 07-07-1897, NP, p. 187.

19. Lettre 67, 14-08-1897, NP, p. 187.

20. Lettre 69, 21-09-1897, NP, p. 190.

21. Lettre 70, 03-10-1897, NP, p. 193. 
22. Lettre 71, 15-10-1897, NP, p. 196.

23. Lettre 75, 15-10-1897, NP, p. 207.

24. O. Mannoni, op. cit., p. 129-130.

25. J. Laplanche, Problématiques V, op. cit., p. 284-285.

26. Lettre 81, 04-01-1898, NP, p. 215.

27. Lettre 83, o9-02-1898, NP, p. 217.

28. D. Anzieu, op. cit., p. 407-459.

29. Lettre $119,21-09-1899, N P$, p. 265 et 266.

30. Lettre 119, 21-09-1899, NP, p. 265 et 266.

31. Lettre 122, 27-10-1899, NP, p. 268.

32. Lettre 131, 23-03-1900, NP, p. 279.

33. Lettre 134, 07-05-1900, NP, p. 283.

34. Lettre 136, 20-05-1900, NP, p. 285.

35. Lettre 138, 10-07-1900, NP, p. 290..

36. Lettre 142, 15-02-1902, NP, p. 291.

37. Lettre 145, 07-08-1901, NP, p. 264-265.

38. Passage inédit de la lettre 145, dans Schur, op. cit., p. 264-265.

39. Texte intégral de Sigmund Freud, publié sous la direction de Joseph Sandler, Paris, Bayard.

40. M. Schur, op. cit., p. 256.

41. J. Laplanche, Le baquet, Transcendance du transfert, Problématique V, Paris, PUF. Pour une information plus complète de cette rupture de Freud, voir notre travail D'où viennent les psychanalystes? Où vont-ils?

42. Voir à ce sujet P. Mahony, 1990, Freud, l'écrivain, Paris, Les Belles Lettres.

43. J. Mazel, 1987, Socrate, Paris, Fayard, p. 32.

44. Elle fut fermée par l'empereur romain Sylla en 87 av. J.-C. Un siècle plus tard, elle fut rouverte par l'empereur romain Marc-Aurèle (121-181 apr. J.-C.), lui-même philosophe.

45. J. De Romilly, Les grands sophistes dans l'Athènes de Périclès, Éditions de Fallois.

46. G. Bastide, 1939, Le moment historique de Socrate, Paris, PUF.; J. Mazel, Socrate, op. cit., p. 87.

47. Platon, République, 337 a, GF Flammarion.

48. P. Hadot, Qu'est-ce que la philosophie antique?, Paris, Folio essais.

49. L. Brisson, 2009, Le Point, numéro hors série, avril.

50. Cette information semble douteuse. Dans la Grèce ancienne, une femme qui devenait veuve ne le restait pas très longtemps. La famille lui trouvait rapidement un tuteur qui était ordinairement un homme de la famille.

51. Diogène Laërce parle d'Ariston comme professeur de lutte.

52. Platon, Lettre VII, 324 b, c, 325 b, c.

53. Platon, discours d'Alcibiade, Le Banquet, 215e.

54. P. Hadot, op. cit., p. 48.

55. Platon, Phédon, présentation et traduction par Monique Dixsaut, Paris, Flammarion.

56. V. Goldschmidt, Les dialogues de Platon, Paris, PUF, 1971.

57. A. Thibaudet, Socrate, CNRS ÉDITIONS.

58. J. F. Mattéi, 2005, De l’indignation, essai, Paris, Éditions La Table Ronde.

59. S. Freud, La création littéraire et le rêve éveillé, in Essais de psychanalyse appliquée, Paris, Gallimard.

60. S. Freud, S., Essais de psychanalyse, p. 16-17, Paris, Payot.

61. J. Mazel, op. cit., p. 540.

62. D. Laërce, op. cit., p. 230.

63. G. Vlastos, Socrate, Ironie et philosophie morale, Paris, Aubier; L.-A. Dorion, Socrate, Paris, PUF. 
64. J.-F. Mattéi, Platon, Que sais-je, PUF, p. 7.

65. Voir plus haut, note 49.

66. T. Ménissier, Eros philosophe, une interprétation philosophique du banquet de Platon, Paris, Éditions Kimé.

67. Ancienne ville d'Asie Mineure, près d'Éphèse.

68. L. Brisson, 2009, Platon amoureux? Le Point Hors-série, Les Grandes Biographies, numéro 2, avril.

69. T. Ménissier, op. cit., p. 71.

70. L. Brisson, Le sexe incertain, Les belles lettres. Voir aussi H. I., Marrou, Histoire de l'Éducation dans l'antiquité, Paris, Seuil ; R. Flacelière, L'amour en Grèce, Paris, Hachette.

71. Platon, La République, traduction d'Émile Chambry, introduction d'Auguste Dies, Paris, Belles Lettres.

72. T. Ménissier, op. cit., p.84.

73. Platon, Théétète, $210 \mathrm{~d}$.

74. T. Ménissier, op. cit., p. 72-74.

75. Après la lecture du livre de D. Jouanna, Aspasie de Milet, il est loisible de se demander si Platon n'a pas imaginé le personnage de Diotime d'après celui d'Aspasie, courtisane de haut rang (hétaïre) qui fut ensuite la conjointe de Périclès. Elle lui donna un fils, Périclès le jeune. Or ce livre nous apprend aussi qu'elle réunissait les grands hommes d'Athènes de l'époque. Dans le Ménexène, Socrate nous dit qu'elle lui a enseigné l'art oratoire. Peutêtre lui a-t-elle aussi enseigné la maïeutique et les choses de l'amour, le seul domaine où il avoue avoir un savoir? Il semble que cette femme intelligente et libre a eu beaucoup d'influence sur la politique de Périclès. Les aristocrates très hostiles à la démocratie ne manquèrent pas de soulever les gens contre Aspasie. On lui fit d'ailleurs un procès, duquel Périclès dût la défendre avec tous les moyens dont il disposait. Quelle influence exerçat-elle sur Socrate? Selon Jouanna, Socrate assistait à ces rencontres intellectuelles où il y avait entre autres Périclès, Protagoras, Anaxagore, Euripide, etc. Il aurait eu avec elle des rencontres personnelles et parfois, il se rendait chez elle avec ses élèves. Platon aurait-il été du nombre des disciples? Ce n'est pas invraisemblable, puisqu'il commença à fréquenter Socrate vers 408 av. J.-C.

76. Le Banquet, présentation et traduction par Luc Brisson, Paris, Flammarion.

77. J.-F. Mattéi, op. cit., p. 113.

78. Platon, Le Banquet, 204 e, traduction Luc Brisson, note 407.

79. Voir à ce sujet W. Jaeger, Paideia, p. 144-145.

80. J. Laplanche et J. B.Pontalis, 1967, Vocabulaire de la psychanalyse, Paris, PUF.

81. Voir à ce sujet la Lettre 67, 14-08-1897, NP, p. 187, et la Lettre 69, 1897, NP p. 190.

82. Maïa en grec veut dire sage-femme, maieutikos : accouchement, maïeutique, la méthode de Socrate.

83. Platon, Le Banquet, présentation et traduction par Luc Brisson, introduction, p. 63.

84. Goethe, Faust, acte 1, Théâtre complet, Paris, Gallimard.

85. F. Gantheret, Moi, Monde, Mots, Paris, Gallimard

86. S. Freud, De la technique psychanalytique, Paris, PUF, p. 50

87. Cette phrase de Kojève est citée par J. Lacan, dans Le transfert, Séminaire 1960-1961.

88. Ceci n'a pas échappé à Luc Brisson : voir son introduction p. 27, note 1.

89. J. Lacan, Le transfert, Séminaire, 1960-1961, chapitre XI.

90. Il en est de même du transfert dans la cure analytique. Il apparaît d'abord toujours comme quelque chose d'inédit à celui qui s'y engage. Il ne peut être saisi et compris que par ceux qui sont passés par là, à commencer par Freud lui-même.

91. Comme le remarque justement Luc Brisson dans la note 554. 
92. J. de Romilly, 1995, Alcibiade, Éditions de Fallois.

93. Gregory Vlastos, op. cit., notes complémentaires, 1.4, Eschine Socraticus, p. 340.

94. J.-F. Mattéi, op. cit., p. 123.

95. P-L., Assoun, 1992, Voyage au pays des Mères, Nouvelle Revue de psychanalyse, $\mathrm{n}^{\circ} 45$. 